\title{
Oscillatory and Asymptotic Properties on a Class of Third Nonlinear Dynamic Equations with Damping Term on Time Scales
}

\author{
Qinghua Feng and Huizeng Qin \\ School of Science, Shandong University of Technology, Zhangzhou Road 12, Zibo 255049, China \\ Correspondence should be addressed to Qinghua Feng; fqhua@sina.com
}

Received 4 March 2013; Revised 10 May 2013; Accepted 10 May 2013

Academic Editor: Narcisa C. Apreutesei

Copyright (c) 2013 Q. Feng and H. Qin. This is an open access article distributed under the Creative Commons Attribution License, which permits unrestricted use, distribution, and reproduction in any medium, provided the original work is properly cited.

We establish some new oscillatory and asymptotic criteria for a class of third-order nonlinear dynamic equations with damping term on time scales. The established results on one hand extend some known results in the literature on the other hand unify continuous and discrete analysis. For illustrating the validity of the established results, we also present some applications for them.

\section{Introduction}

The theory of time scale, which was initiated by Hilger [1], trying to treat continuous and discrete analysis in a consistent way, has received a lot of attention in recent years. Various investigations have been done by many authors. Among these investigations, some authors have taken research in the oscillatory and asymptotic properties of dynamic equations on time scales, and there has been increasing interest in obtaining sufficient conditions for the oscillation and asymptotic behavior of solutions of various dynamic equations on time scales (e.g., we refer the reader to [2-20]). But we notice that most of the investigations are concerned with oscillatory and asymptotic properties of solutions of first- or second-order dynamic equations on time scales, while relatively less attention has been paid to oscillatory and asymptotic properties of third-order dynamic equations on time scales. For recent results about the oscillation and asymptotic behavior of solutions of third-order dynamic equations on time scales, we refer the reader to [21-33]. In $[34,35]$, Saker researched oscillation of the following thirdorder dynamic equations:

$$
\left(p(t)\left[\left(r(t) x^{\Delta}(t)\right)^{\Delta}\right]^{\gamma}\right)^{\Delta}+q(t) f(x(\tau(t)))=0 .
$$

Based on the Riccati substitution and the analysis of the associated Riccati dynamic inequality, some new sufficient oscillatory conditions were presented.
Moreover, to our best knowledge, none of the existing results deal with oscillatory and asymptotic behavior of solutions of third-order nonlinear dynamic equations with damping term on time scales, in which the damping term brings new difficulty in obtaining oscillatory and asymptotic criteria. We now list some important results.

In this paper, we are concerned with oscillatory and asymptotic behavior of solutions of the third-order nonlinear dynamic equation with damping term on time scales of the following form:

$$
\begin{aligned}
& \left(a(t)\left(\left[r(t) x^{\Delta}(t)\right]^{\Delta}\right)^{\gamma}\right)^{\Delta} \\
& \quad+p(t)\left(\left[r(t) x^{\Delta}(t)\right]^{\Delta}\right)^{\gamma}+q(t) f(x(t))=0, \quad t \in \mathbb{T}_{0},
\end{aligned}
$$

where $\mathbb{T}$ is an arbitrary time scale, $\mathbb{T}_{0}=\left[t_{0}, \infty\right) \bigcap \mathbb{T}, a, r$, $p, q \in C_{\mathrm{rd}}\left(\mathbb{T}_{0}, \mathbb{R}_{+}\right), f \in C(\mathbb{R}, \mathbb{R})$ satisfying $x f(x)>$ $0, f(x) / x^{\gamma} \geq L>0$ for $x \neq 0$, and $\gamma \geq 1$ is a quotient of two odd positive integers.

A solution of (2) is said to be oscillatory if it is neither eventually positive nor eventually negative otherwise it is nonoscillatory. Equation (2) is said to be oscillatory in case all its solutions are oscillatory.

We will establish some new criteria of oscillatory and asymptotic behavior for (2) by a generalized Riccati transformation technique in Section 2 and present some applications 
for our results in Section 3. Throughout this paper, $\mathbb{R}$ denotes the set of real numbers and $\mathbb{R}_{+}=(0, \infty)$, while $\mathbb{Z}$ denotes the set of integers. $\mathbb{T}$ denotes an arbitrary time scale and $t_{i} \in \mathbb{T}, i=$ $1,2, \ldots, 5$. On $\mathbb{T}$ we define the forward and backward jump operators $\sigma \in(\mathbb{T}, \mathbb{T})$ and $\rho \in(\mathbb{T}, \mathbb{T})$ such that $\sigma(t)=\inf \{s \in$ $\mathbb{T}, s>t\}, \rho(t)=\sup \{s \in \mathbb{T}, s<t\}$. A point $t \in \mathbb{T}$ with $t>\inf \mathbb{T}$ is said to be left-dense if $\rho(t)=t$, right-dense if $\sigma(t)=t$, leftscattered if $\rho(t)<t$, and right-scattered if $\sigma(t)>t$. A function $f \in(\mathbb{T}, \mathbb{R})$ is called rd-continuous if it is continuous in rightdense points and if the left-sided limits exist in left-dense points, while $f$ is called regressive if $1+\mu(t) f(t) \neq 0$, where $\mu(t)=\sigma(t)-t . C_{\text {rd }}$ denotes the set of rd-continuous functions, while $\mathfrak{R}$ denotes the set of all regressive and rd-continuous functions, and $\mathfrak{R}^{+}=\{f \mid f \in \mathfrak{R}, 1+\mu(t) f(t)>0, \forall t \in \mathbb{T}\}$.

Definition 1. For $p \in \mathfrak{R}$, the exponential function is defined by

$$
e_{p}(t, s)=\exp \left(\int_{s}^{t} \xi_{\mu(\tau)}(p(\tau)) \Delta \tau\right) \quad \text { for } s, t \in \mathbb{T} .
$$

Remark 2. If $\mathbb{T}=\mathbb{R}$, then

$$
e_{p}(t, s)=\exp \left(\int_{s}^{t} p(\tau) d \tau\right), \quad \text { for } s, t \in \mathbb{R} .
$$

If $\mathbb{T}=\mathbb{Z}$, then

$$
e_{p}(t, s)=\prod_{\tau=s}^{t-1}[1+p(\tau)], \quad \text { for } s, t \in \mathbb{Z}, s<t .
$$

The following two theorems include some known properties on the exponential function.

Theorem 3 (see [36, Theorem 5.1]). If $p \in \mathfrak{R}$ and fix $t_{0} \in \mathbb{T}$, then the exponential function $e_{p}\left(t, t_{0}\right)$ is the unique solution of the following initial value problem

$$
\begin{gathered}
y^{\Delta}(t)=p(t) y(t), \\
y\left(t_{0}\right)=1 .
\end{gathered}
$$

Theorem 4 (see [36, Theorem 5.2]). If $p \in \mathfrak{R}^{+}$, then $e_{p}(t, s)>$ 0 for $\forall s, t \in \mathbb{T}$.

For more details about the calculus of time scales, we refer to [37].

\section{Main Results}

For the sake of convenience, in the rest of this paper, we set $\delta_{1}(t, a)=\int_{a}^{t}\left(\left[e_{-p / a}\left(s, t_{0}\right)\right]^{1 / \gamma} / a^{1 / \gamma}(s)\right) \Delta s, \delta_{2}(t, a)=$ $\int_{a}^{t}\left(\delta_{1}(s, a) / r(s)\right) \Delta s$.

Lemma 5. Suppose $-p / a \in \mathfrak{R}_{+}$, and assume that

$$
\begin{gathered}
\int_{t_{0}}^{\infty} \frac{\left[e_{-p / a}\left(s, t_{0}\right)\right]^{1 / \gamma}}{a^{1 / \gamma}(s)} \Delta s=\infty, \\
\int_{t_{0}}^{\infty} \frac{1}{r(s)} \Delta s=\infty
\end{gathered}
$$

and (2) has an eventually positive solution $x$. Then there exists a sufficiently large $T_{1}^{*} \in \mathbb{U}$ such that

$$
\begin{aligned}
& \left(\frac{a(t)\left(\left[r(t) x^{\Delta}(t)\right]^{\Delta}\right)^{\gamma}}{e_{-p / a}\left(t, t_{0}\right)}\right)^{\Delta}<0, \\
& {\left[r(t) x^{\Delta}(t)\right]^{\Delta}>0 \text { on }\left[T_{1}^{*}, \infty\right)_{\mathbb{T}} .}
\end{aligned}
$$

Proof. By $-p / a \in \mathfrak{R}_{+}$, we have $e_{-p / a}\left(t, t_{0}\right)>0$. Since $x$ is eventually a positive solution of (2), there exists a sufficiently large $t_{1}$ such that $x(t)>0$ on $\left[t_{1}, \infty\right)_{\mathbb{V}}$, and for $t \in\left[t_{1}, \infty\right)_{\mathbb{V}}$, we obtain that

$$
\begin{aligned}
& \left(\frac{a(t)\left(\left[r(t) x^{\Delta}(t)\right]^{\Delta}\right)^{\gamma}}{e_{-p / a}\left(t, t_{0}\right)}\right)^{\Delta} \\
& =\left(e_{-p / a}\left(t, t_{0}\right)\left(a(t)\left(\left[r(t) x^{\Delta}(t)\right]^{\Delta}\right)^{\gamma}\right)^{\Delta}\right. \\
& \quad \times\left(e_{-p / a}\left(t, t_{0}\right) e_{-p / a}\left(\sigma(t), t_{0}\right)\right)^{-1} \\
& =\frac{\left.-\left(e_{-p / a}\left(t, t_{0}\right)\right)^{\Delta} a(t)\left(\left[r(t) x^{\Delta}(t)\right]^{\Delta}\right)^{\gamma}\right)}{\left(a(t)\left(\left[r(t) x^{\Delta}(t)\right]^{\Delta}\right)^{\gamma}\right)^{\Delta}+p(t)\left(\left[r(t) x^{\Delta}(t)\right]^{\Delta}\right)^{\gamma}} \\
& =\frac{-q(t) f(x(t))}{e_{-p / a}\left(\sigma(t), t_{0}\right)}<0 .
\end{aligned}
$$

Then $a(t)\left(\left[r(t) x^{\Delta}(t)\right]^{\Delta}\right)^{\gamma} / e_{-p / a}\left(t, t_{0}\right)$ is strictly decreasing on $\left[t_{1}, \infty\right)_{\mathbb{T}}$, and together with $a(t)>0, e_{-p / a}\left(t, t_{0}\right)>0$ we deduce that $\left[r(t) x^{\Delta}(t)\right]^{\Delta}$ is eventually of one sign. We claim $\left[r(t) x^{\Delta}(t)\right]^{\Delta}>0$ on $\left[t_{2}, \infty\right)_{\mathbb{T}}$. Otherwise, assume there exists a sufficiently large $t_{3}$ such that $\left[r(t) x^{\Delta}(t)\right]^{\Delta}<0$ on $\left[t_{3}, \infty\right)_{\mathbb{T}}$. Then

$$
\begin{aligned}
r(t) & x^{\Delta}(t)-r\left(t_{3}\right) x^{\Delta}\left(t_{3}\right) \\
= & \int_{t_{3}}^{t} \frac{\left[e_{-p / a}\left(s, t_{0}\right) a(s)\right]^{1 / \gamma}\left[r(s) x^{\Delta}(s)\right]^{\Delta}}{\left[e_{-p / a}\left(s, t_{0}\right) a(s)\right]^{1 / \gamma}} \Delta s \\
& \leq \frac{a^{1 / \gamma}\left(t_{3}\right)\left[r\left(t_{3}\right) x^{\Delta}\left(t_{3}\right)\right]^{\Delta}}{\left[e_{-p / a}\left(t_{3}, t_{0}\right)\right]^{1 / \gamma}} \int_{t_{3}}^{t} \frac{\left[e_{-p / a}\left(s, t_{0}\right)\right]^{1 / \gamma}}{a^{1 / \gamma}(s)} \Delta s .
\end{aligned}
$$

By (7), we have $\lim _{t \rightarrow \infty} r(t) x^{\Delta}(t)=-\infty$, and thus there exists a sufficiently large $t_{4} \in\left[t_{3}, \infty\right)_{\mathbb{T}}$ such that $r(t) x^{\Delta}(t)<0$ on $\left[t_{4}, \infty\right)_{\mathbb{T}}$. By the assumption $\left[r(t) x^{\Delta}(t)\right]^{\Delta}<0$ one can see $r(t) x^{\Delta}(t)$ is strictly decreasing on $\left[t_{4}, \infty\right)_{\mathbb{T}}$, and then

$$
x(t)-x\left(t_{4}\right)=\int_{t_{4}}^{t} \frac{r(s) x^{\Delta}(s)}{r(s)} \Delta s \leq r\left(t_{4}\right) x^{\Delta}\left(t_{4}\right) \int_{t_{4}}^{t} \frac{1}{r(s)} \Delta s .
$$


Using (8), we have $\lim _{t \rightarrow \infty} x(t)=-\infty$, which leads to a contradiction. So $\left[r(t) x^{\Delta}(t)\right]^{\Delta}>0$ on $\left[t_{2}, \infty\right)_{\mathbb{T}}$, and the proof is complete with taking $T_{1}^{*}=t_{2}$.

Lemma 6. Under the conditions of Lemma 5, furthermore, assume that

$$
\begin{aligned}
\lim _{t \rightarrow \infty} \sup \int_{t_{0}}^{t}[ & \frac{1}{r(\xi)} \\
& \quad \times \int_{\xi}^{\infty}\left(\frac{e_{-p / a}\left(\tau, t_{0}\right)}{a(\tau)}\right. \\
& \left.\left.\quad \times \int_{\tau}^{\infty} \frac{q(s)}{e_{-p / a}\left(\sigma(s), t_{0}\right)} \Delta s\right)^{1 / \gamma} \Delta \tau\right] \Delta \xi
\end{aligned}
$$

$=\infty$.

Then either there exists a sufficiently large $T_{2}^{*} \in \mathbb{T}$ such that $x^{\Delta}(t)>0$ on $\left[T_{2}^{*}, \infty\right)_{\mathbb{T}}$ or $\lim _{t \rightarrow \infty} x(t)=0$.

Proof. By Lemma 5, we deduce that $x^{\Delta}(t)$ is eventually of one sign. So there exists a sufficiently large $t_{5}>t_{4}$ such that either $x^{\Delta}(t)>0$ or $x^{\Delta}(t)<0$ on $\left[t_{5}, \infty\right)_{\mathbb{T}}$, where $t_{4}$ is defined as in Lemma 5. If $x^{\Delta}(t)<0$, together with $x(t)$ is an eventually positive solution of (2), we obtain $\lim _{t \rightarrow \infty} x(t)=\alpha \geq 0$ and $\lim _{t \rightarrow \infty} r(t) x^{\Delta}(t)=\beta \leq 0$. We claim $\alpha=0$. Otherwise, assume $\alpha>0$. Then $x(t) \geq \alpha$ on $\left[t_{5}, \infty\right)_{\mathbb{T}}$, and for $t \in$ $\left[t_{5}, \infty\right) \cap \mathbb{T}$, an integration for (10) from $t$ to $\infty$ yields

$$
\begin{gathered}
-\frac{a(t)\left(\left[r(t) x^{\Delta}(t)\right]^{\Delta}\right)^{\gamma}}{e_{-p / a}\left(t, t_{0}\right)} \\
=-\lim _{t \rightarrow \infty} \frac{a(t)\left(\left[r(t) x^{\Delta}(t)\right]^{\Delta}\right)^{\gamma}}{e_{-p / a}\left(t, t_{0}\right)} \\
+\int_{t}^{\infty} \frac{-q(s) f(x(s))}{e_{-p / a}\left(\sigma(s), t_{0}\right)} \Delta s \\
\leq-\lim _{t \rightarrow \infty} \frac{a(t)\left(\left[r(t) x^{\Delta}(t)\right]^{\Delta}\right)^{\gamma}}{e_{-p / a}\left(t, t_{0}\right)} \\
+\int_{t}^{\infty} \frac{-L q(s) x^{\gamma}(s)}{e_{-p / a}\left(\sigma(s), t_{0}\right)} \Delta s \\
\leq-L \int_{t}^{\infty} \frac{q(s) x^{\gamma}(s)}{e_{-p / a}\left(\sigma(s), t_{0}\right)} \Delta s \\
\leq-L \alpha^{\gamma} \int_{t}^{\infty} \frac{q(s)}{e_{-p / a}\left(\sigma(s), t_{0}\right)} \Delta s,
\end{gathered}
$$

which is followed by

$$
\begin{aligned}
& -\left[r(t) x^{\Delta}(t)\right]^{\Delta} \\
& \leq-\left\{L \alpha^{\gamma}\left[\frac{e_{-p / a}\left(t, t_{0}\right)}{a(t)} \int_{t}^{\infty} \frac{q(s)}{e_{-p / a}\left(\sigma(s), t_{0}\right)} \Delta s\right]\right\}^{1 / \gamma} .
\end{aligned}
$$

Substituting $t$ with $\tau$ in (15), an integration for (15) with respect to $\tau$ from $t$ to $\infty$ yields

$$
\begin{aligned}
& r(t) x^{\Delta}(t) \\
& =\lim _{t \rightarrow \infty} r(t) x^{\Delta}(t)-\alpha L^{1 / \gamma} \\
& \quad \times \int_{t}^{\infty}\left(\frac{e_{-p / a}\left(\tau, t_{0}\right)}{a(\tau)} \int_{\tau}^{\infty} \frac{q(s)}{e_{-p / a}\left(\sigma(s), t_{0}\right)} \Delta s\right)^{1 / \gamma} \Delta \tau \\
& =\beta-\alpha L^{1 / \gamma} \int_{t}^{\infty}\left(\frac{e_{-p / a}\left(\tau, t_{0}\right)}{a(\tau)} \int_{\tau}^{\infty} \frac{q(s)}{e_{-p / a}\left(\sigma(s), t_{0}\right)} \Delta s\right)^{1 / \gamma} \Delta \tau \\
& \leq-\alpha L^{1 / \gamma} \int_{t}^{\infty}\left(\frac{e_{-p / a}\left(\tau, t_{0}\right)}{a(\tau)} \int_{\tau}^{\infty} \frac{q(s)}{e_{-p / a}\left(\sigma(s), t_{0}\right)} \Delta s\right)^{1 / \gamma} \Delta \tau,
\end{aligned}
$$

which implies

$$
\begin{aligned}
& x^{\Delta}(t) \\
& \leq-\alpha L^{1 / \gamma} \frac{1}{r(t)} \int_{t}^{\infty}\left(\frac{e_{-p / a}\left(\tau, t_{0}\right)}{a(\tau)}\right. \\
& \left.\quad \times \int_{\tau}^{\infty} \frac{q(s)}{e_{-p / a}\left(\sigma(s), t_{0}\right)} \Delta s\right)^{1 / \gamma} \Delta \tau .
\end{aligned}
$$

Substituting $t$ with $\xi$ in (17), an integration for (17) with respect to $\xi$ from $t_{5}$ to $t$ yields

$$
\begin{aligned}
x(t)-x\left(t_{5}\right) & \\
\leq-\alpha L^{1 / \gamma} \int_{t_{5}}^{t}[ & \frac{1}{r(\xi)} \\
& \times \int_{\xi}^{\infty}\left(\frac{e_{-p / a}\left(\tau, t_{0}\right)}{a(\tau)}\right. \\
& \left.\left.\times \int_{\tau}^{\infty} \frac{q(s)}{e_{-p / a}\left(\sigma(s), t_{0}\right)} \Delta s\right)^{1 / \gamma} \Delta \tau\right] \Delta \xi .
\end{aligned}
$$

By (18) and (13) we have $\lim _{t \rightarrow \infty} x(t)=-\infty$, which leads to a contradiction. So one has $\alpha=0$, and the proof is complete. 
Lemma 7. Suppose $-p / a \in \mathfrak{R}_{+}$, and assume that $x$ is a positive solution of (2) such that

$$
\left[r(t) x^{\Delta}(t)\right]^{\Delta}>0, \quad x^{\Delta}(t)>0 \text { on }\left[T_{3}^{*}, \infty\right)_{\mathbb{T}},
$$

where $T_{3}^{*} \in \mathbb{T}$ is sufficiently large. Then for $t \in\left[T_{3}^{*}, \infty\right)_{\mathbb{T}}$, we have

$$
\begin{aligned}
& x^{\Delta}(t) \geq \frac{\delta_{1}\left(t, T_{3}^{*}\right)}{r(t)}\left\{\frac{a^{1 / \gamma}(t)\left[r(t) x^{\Delta}(t)\right]^{\Delta}}{\left[e_{-p / a}\left(t, t_{0}\right)\right]^{1 / \gamma}}\right\}, \\
& x(t) \geq \delta_{2}\left(t, T_{3}^{*}\right)\left\{\frac{a^{1 / \gamma}(t)\left[r(t) x^{\Delta}(t)\right]^{\Delta}}{\left[e_{-p / a}\left(t, t_{0}\right)\right]^{1 / \gamma}}\right\} .
\end{aligned}
$$

Proof. Take $T_{3}^{*}>\max \left(T_{1}^{*}, T_{2}^{*}\right)$, where $T_{1}^{*}, T_{2}^{*}$ are defined as in Lemmas 5 and 6 , respectively. By Lemma 5 we have $a(t)$ $\left(\left[r(t) x^{\Delta}(t)\right]^{\Delta}\right)^{\gamma} / e_{-p / a}\left(t, t_{0}\right)$ strictly decreasing on $\left[T_{3}^{*}, \infty\right)$. So

$$
\begin{aligned}
r(t) x^{\Delta}(t) \geq & r(t) x^{\Delta}(t)-r\left(T_{3}^{*}\right) x^{\Delta}\left(T_{3}^{*}\right) \\
= & \int_{T_{3}^{*}}^{t} \frac{\left[e_{-p / a}\left(s, t_{0}\right) a(s)\right]^{1 / \gamma}\left[r(s) x^{\Delta}(s)\right]^{\Delta}}{\left[e_{-p / a}\left(s, t_{0}\right) a(s)\right]^{1 / \gamma}} \Delta s \\
\geq & \frac{a^{1 / \gamma}(t)\left[r(t) x^{\Delta}(t)\right]^{\Delta}}{\left[e_{-p / a}\left(t, t_{0}\right)\right]^{1 / \gamma}} \\
& \times \int_{T_{3}^{*}}^{t} \frac{\left[e_{-p / a}\left(s, t_{0}\right)\right]^{1 / \gamma}}{a^{1 / \gamma}(s)} \Delta s \\
= & \delta_{1}\left(t, T_{3}^{*}\right) \frac{a^{1 / \gamma}(t)\left[r(t) x^{\Delta}(t)\right]^{\Delta}}{\left[e_{-p / a}\left(t, t_{0}\right)\right]^{1 / \gamma}}
\end{aligned}
$$

and then

$$
x^{\Delta}(t) \geq \frac{\delta_{1}\left(t, T_{3}^{*}\right)}{r(t)}\left\{\frac{a^{1 / \gamma}(t)\left[r(t) x^{\Delta}(t)\right]^{\Delta}}{\left[e_{-p / a}\left(t, t_{0}\right)\right]^{1 / \gamma}}\right\} .
$$

Furthermore,

$$
\begin{aligned}
x(t) \geq & x(t)-x\left(T_{3}^{*}\right) \\
= & \int_{T_{3}^{*}}^{t} x^{\Delta}(s) \Delta s \\
\geq & \int_{T_{3}^{*}}^{t} \frac{\delta_{1}\left(s, T_{3}^{*}\right)}{r(s)}\left\{\frac{a^{1 / \gamma}(s)\left[r(s) x^{\Delta}(s)\right]^{\Delta}}{\left[e_{-p / a}\left(s, t_{0}\right)\right]^{1 / \gamma}}\right\} \Delta s \\
\geq & \left\{\frac{a^{1 / \gamma}(t)\left[r(t) x^{\Delta}(t)\right]^{\Delta}}{\left[e_{-p / a}\left(t, t_{0}\right)\right]^{1 / \gamma}}\right\} \\
& \times \int_{T_{3}^{*}}^{t} \frac{\delta_{1}\left(s, T_{3}^{*}\right)}{r(s)} \Delta s \\
= & \delta_{2}\left(t, T_{3}^{*}\right)\left\{\frac{a^{1 / \gamma}(t)\left[r(t) x^{\Delta}(t)\right]^{\Delta}}{\left[e_{-p / a}\left(t, t_{0}\right)\right]^{1 / \gamma}}\right\},
\end{aligned}
$$

which is the desired result.

Lemma 8 (see [38, Theorem 41]). Assume that $X$ and $Y$ are nonnegative real numbers. Then

$$
\lambda X Y^{\lambda-1}-X^{\lambda} \leq(\lambda-1) Y^{\lambda} \quad \forall \lambda>1 .
$$

Theorem 9. Suppose $-p / a \in \mathfrak{R}_{+}$, and assume that (7), (8), and (13) hold, and for all sufficiently large $T$,

$$
\begin{aligned}
\lim _{t \rightarrow \infty} \sup \left\{\int _ { T } ^ { t } \left\{L \frac{q(s) \rho(s)}{e_{-p / a}\left(\sigma(s), t_{0}\right)}-\rho(t)[a(s) \eta(s)]^{\Delta}+\frac{\rho(s) \delta_{1}(s, T)[a(\sigma(s)) \eta(\sigma(s))]^{1+1 / \gamma}}{r(s)}\right.\right. \\
\left.\left.-\left[\frac{r(s) \rho^{\Delta}(s)+(\gamma+1) \rho(s) \delta_{1}(s, T)[a(\sigma(s)) \eta(\sigma(s))]^{1 / \gamma}}{(\gamma+1) r^{1 /(\gamma+1)}(s) \rho^{\gamma /(\gamma+1)}(s) \delta_{1}^{\gamma /(\gamma+1)}(s, T)}\right]^{\gamma+1}\right\} \Delta s\right\}=\infty,
\end{aligned}
$$

where $\rho, \eta$ are two given nonnegative functions on $\mathbb{T}$ with $\rho(t)>0$. Then every solution of $(2)$ is oscillatory or tends to zero.

Proof. Assume (2) has a nonoscillatory solution $x$ on $\mathbb{T}_{0}$. Without loss of generality, we may assume $x(t)>0$ on $\left[t_{1}, \infty\right)_{\mathbb{T}}$, where $t_{1}$ is sufficiently large. By Lemmas 5 and 6 , there exists sufficiently large $t_{2}$ such that $\left[r(t) x^{\Delta}(t)\right]^{\Delta}>$ 0 on $\left[t_{2}, \infty\right)_{\mathbb{T}}$, and either $x^{\Delta}(t)>0$ on $\left[t_{2}, \infty\right)_{\mathbb{T}}$ or $\lim _{t \rightarrow \infty} x(t)=0$. Now we assume $x^{\Delta}(t)>0$ on $\left[t_{2}, \infty\right)_{\mathbb{T}}$. Define the generalized Riccati function:

$$
\omega(t)=\rho(t) a(t)\left[\frac{\left(\left[r(t) x^{\Delta}(t)\right]^{\Delta}\right)^{\gamma}}{x^{\gamma}(t) e_{-p / a}\left(t, t_{0}\right)}+\eta(t)\right] .
$$


Then for $t \in\left[t_{2}, \infty\right)_{\mathbb{T}}$, we have

$\omega^{\Delta}(t)$

$$
\begin{aligned}
& =\frac{\rho(t)}{x^{\gamma}(t)}\left\{\frac{a(t)\left(\left[r(t) x^{\Delta}(t)\right]^{\Delta}\right)^{\gamma}}{e_{-p / a}\left(t, t_{0}\right)}\right\}^{\Delta}+\left[\frac{\rho(t)}{x^{\gamma}(t)}\right]^{\Delta} \\
& \quad \times \frac{a(\sigma(t))\left(\left[r(\sigma(t)) x^{\Delta}(\sigma(t))\right]^{\Delta}\right)^{\gamma}}{e_{-p / a}\left(\sigma(t), t_{0}\right)}
\end{aligned}
$$

$+\rho(t)[a(t) \eta(t)]^{\Delta}+\rho^{\Delta}(t) a(\sigma(t)) \eta(\sigma(t))$

$=\frac{\rho(t)}{x^{\gamma}(t)}$

$$
\times\left\{\left(e_{-p / a}\left(t, t_{0}\right)\left(a(t)\left(\left[r(t) x^{\Delta}(t)\right]^{\Delta}\right)^{\gamma}\right)^{\Delta}\right.\right.
$$$$
\left.-\left(e_{-p / a}\left(t, t_{0}\right)\right)^{\Delta} a(t)\left(\left[r(t) x^{\Delta}(t)\right]^{\Delta}\right)^{\gamma}\right)
$$$$
\left.\times\left(e_{-p / a}\left(t, t_{0}\right) e_{-p / a}\left(\sigma(t), t_{0}\right)\right)^{-1}\right\}
$$$$
+\left[\frac{x^{\gamma}(t) \rho^{\Delta}(t)-\left(x^{\gamma}(t)\right)^{\Delta} \rho(t)}{x^{\gamma}(t) x^{\gamma}(\sigma(t))}\right]
$$$$
\times \frac{a(\sigma(t))\left(\left[r(\sigma(t)) x^{\Delta}(\sigma(t))\right]^{\Delta}\right)^{\gamma}}{e_{-p / a}\left(\sigma(t), t_{0}\right)}
$$

$+\rho(t)[a(t) \eta(t)]^{\Delta}+\rho^{\Delta}(t) a(\sigma(t)) \eta(\sigma(t))$

$$
\begin{aligned}
& =\frac{\rho(t)}{x^{\gamma}(t)} \\
& \times\left[\left(\left(a(t)\left(\left[r(t) x^{\Delta}(t)\right]^{\Delta}\right)^{\gamma}\right)^{\Delta}\right.\right. \\
& \left.+p(t)\left(\left[r(t) x^{\Delta}(t)\right]^{\Delta}\right)^{\gamma}\right) \\
& \left.\times\left(e_{-p / a}\left(\sigma(t), t_{0}\right)\right)^{-1}\right] \\
& +\frac{\rho^{\Delta}(t)}{\rho(\sigma(t))} \omega(\sigma(t))-\left[\frac{\rho(t)\left(x^{\gamma}(t)\right)^{\Delta}}{x^{\gamma}(t)}\right] \\
& \times \frac{a(\sigma(t))\left(\left[r(\sigma(t)) x^{\Delta}(\sigma(t))\right]^{\Delta}\right)^{\gamma}}{x^{\gamma}(\sigma(t)) e_{-p / a}\left(\sigma(t), t_{0}\right)} \\
& +\rho(t)[a(t) \eta(t)]^{\Delta} \\
& =-\frac{\rho(t)}{x^{\gamma}(t)}\left[\frac{q(t) f(x(t))}{e_{-p / a}\left(\sigma(t), t_{0}\right)}\right]+\frac{\rho^{\Delta}(t)}{\rho(\sigma(t))} \omega(\sigma(t)) \\
& -\left[\frac{\rho(t)\left(x^{\gamma}(t)\right)^{\Delta}}{x^{\gamma}(t)}\right] \frac{a(\sigma(t))\left(\left[r(\sigma(t)) x^{\Delta}(\sigma(t))\right]^{\Delta}\right)^{\gamma}}{x^{\gamma}(\sigma(t)) e_{-p / a}\left(\sigma(t), t_{0}\right)}
\end{aligned}
$$

$$
\begin{aligned}
& +\rho(t)[a(t) \eta(t)]^{\Delta} \\
\leq & -L \frac{q(t) \rho(t)}{e_{-p / a}\left(\sigma(t), t_{0}\right)}+\frac{\rho^{\Delta}(t)}{\rho(\sigma(t))} \omega(\sigma(t)) \\
& -\left[\frac{\rho(t)\left(x^{\gamma}(t)\right)^{\Delta}}{x^{\gamma}(t)}\right] \\
& \times \frac{a(\sigma(t))\left(\left[r(\sigma(t)) x^{\Delta}(\sigma(t))\right]^{\Delta}\right)^{\gamma}}{x^{\gamma}(\sigma(t)) e_{-p / a}\left(\sigma(t), t_{0}\right)} \\
& +\rho(t)[a(t) \eta(t)]^{\Delta} .
\end{aligned}
$$

By [37, Theorem 1.93], we have $\left(x^{\gamma}(t)\right)^{\Delta} \geq \gamma x^{\gamma-1}(t) x^{\Delta}(t)$. Then

$$
\begin{aligned}
& \omega^{\Delta}(t) \leq-L \frac{q(t) \rho(t)}{e_{-p / a}\left(\sigma(t), t_{0}\right)}+\frac{\rho^{\Delta}(t)}{\rho(\sigma(t))} \omega(\sigma(t)) \\
& -\rho(t)\left[\frac{\gamma x^{\gamma-1}(t) x^{\Delta}(t)}{x^{\gamma}(t)}\right] \\
& \times \frac{a(\sigma(t))\left(\left[r(\sigma(t)) x^{\Delta}(\sigma(t))\right]^{\Delta}\right)^{\gamma}}{x^{\gamma}(\sigma(t)) e_{-p / a}\left(\sigma(t), t_{0}\right)} \\
& +\rho(t)[a(t) \eta(t)]^{\Delta} \\
& \leq-L \frac{q(t) \rho(t)}{e_{-p / a}\left(\sigma(t), t_{0}\right)}+\frac{\rho^{\Delta}(t)}{\rho(\sigma(t))} \omega(\sigma(t)) \\
& -\left[\frac{\gamma \rho(t)}{x(\sigma(t))}\right] \\
& \times\left\{\frac{\delta_{1}\left(t, t_{2}\right)}{r(t)}\left[\frac{a^{1 / \gamma}(t)\left[r(t) x^{\Delta}(t)\right]^{\Delta}}{\left[e_{-p / a}\left(t, t_{0}\right)\right]^{1 / \gamma}}\right]\right\} \\
& \times \frac{a(\sigma(t))\left(\left[r(\sigma(t)) x^{\Delta}(\sigma(t))\right]^{\Delta}\right)^{\gamma}}{x^{\gamma}(\sigma(t)) e_{-p / a}\left(\sigma(t), t_{0}\right)} \\
& +\rho(t)[a(t) \eta(t)]^{\Delta} \\
& =-L \frac{q(t) \rho(t)}{e_{-p / a}\left(\sigma(t), t_{0}\right)}+\frac{\rho^{\Delta}(t)}{\rho(\sigma(t))} \omega(\sigma(t)) \\
& -\gamma \frac{\rho(t) \delta_{1}\left(t, t_{2}\right)}{r(t)} \\
& \times\left[\frac{\omega(\sigma(t))}{\rho(\sigma(t))}-a(\sigma(t)) \eta(\sigma(t))\right]^{1+1 / \gamma} \\
& +\rho(t)[a(t) \eta(t)]^{\Delta} .
\end{aligned}
$$

Using the following inequality (see [25, (2.17)]):

$$
(u-v)^{1+1 / \gamma} \geq u^{1+1 / \gamma}+\frac{1}{\gamma} v^{1+1 / \gamma}-\left(1+\frac{1}{\gamma}\right) v^{1 / \gamma} u,
$$


where $u, v$ are constants and $\gamma \geq 1$ is a quotient of two odd positive integers, we obtain

$$
\begin{aligned}
& {\left[\frac{\omega(\sigma(t))}{\rho(\sigma(t))}-a(\sigma(t)) \eta(\sigma(t))\right]^{1+1 / \gamma}} \\
& \geq \frac{\omega^{1+1 / \gamma}(\sigma(t))}{\rho^{1+1 / \gamma}(\sigma(t))}+\frac{1}{\gamma}[a(\sigma(t)) \eta(\sigma(t))]^{1+1 / \gamma} \\
& \quad-\left(1+\frac{1}{\gamma}\right) \frac{[a(\sigma(t)) \eta(\sigma(t))]^{1 / \gamma} \omega(\sigma(t))}{\rho(\sigma(t))} .
\end{aligned}
$$

A combination of (28) and (30) yields

$$
\begin{aligned}
& \omega^{\Delta}(t) \\
& \leq-L \frac{q(t) \rho(t)}{e_{-p / a}\left(\sigma(t), t_{0}\right)}+\rho(t)[a(t) \eta(t)]^{\Delta} \\
& \quad-\frac{\rho(t) \delta_{1}\left(t, t_{2}\right)[a(\sigma(t)) \eta(\sigma(t))]^{1+1 / \gamma}}{r(t)} \\
& +\frac{r(t) \rho^{\Delta}(t)+(\gamma+1) \rho(t) \delta_{1}\left(t, t_{2}\right)[a(\sigma(t)) \eta(\sigma(t))]^{1 / \gamma}}{r(t) \rho(\sigma(t))} \\
& \quad \times \omega(\sigma(t))-\gamma \frac{\rho(t) \delta_{1}\left(t, t_{2}\right)}{r(t)} \frac{\omega^{1+1 / \gamma}(\sigma(t))}{\rho^{1+1 / \gamma}(\sigma(t))} .
\end{aligned}
$$

Setting

$$
\begin{gathered}
\lambda=1+\frac{1}{\gamma}, \\
X^{\lambda}=\gamma \frac{\rho(t) \delta_{1}\left(t, t_{2}\right)}{r(t)} \frac{\omega^{1+1 / \gamma}(\sigma(t))}{\rho^{1+1 / \gamma}(\sigma(t))}, \\
Y^{\lambda-1} \\
\gamma^{1 /(\gamma+1)} \times\left[\frac{r(t) \rho^{\Delta}(t)+(\gamma+1) \rho(t) \delta_{1}\left(t, t_{2}\right)[a(\sigma(t)) \eta(\sigma(t))]^{1 / \gamma}}{(\gamma+1) r^{1 /(\gamma+1)}(t) \rho^{\gamma /(\gamma+1)}(t) \delta_{1}^{\gamma /(\gamma+1)}\left(t, t_{2}\right)}\right] .
\end{gathered}
$$

Using Lemma 8 in (31) we get that

$$
\begin{aligned}
& \omega^{\Delta}(t) \\
& \leq-L \frac{q(t) \rho(t)}{e_{-p / a}\left(\sigma(t), t_{0}\right)}+\rho(t)[a(t) \eta(t)]^{\Delta} \\
& \quad-\frac{\rho(t) \delta_{1}\left(t, t_{2}\right)[a(\sigma(t)) \eta(\sigma(t))]^{1+1 / \gamma}}{r(t)} \\
& +\left[\frac{r(t) \rho^{\Delta}(t)+(\gamma+1) \rho(t) \delta_{1}\left(t, t_{2}\right)[a(\sigma(t)) \eta(\sigma(t))]^{1 / \gamma}}{(\gamma+1) r^{1 /(\gamma+1)}(t) \rho^{\gamma /(\gamma+1)}(t) \delta_{1}^{\gamma /(\gamma+1)}\left(t, t_{2}\right)}\right]^{\gamma+1}
\end{aligned}
$$

Substituting $t$ with $s$ in (33), an integration for (33) with respect to $s$ from $t_{2}$ to $t$ yields

$$
\begin{aligned}
\int_{t_{2}}^{t}\left\{L \frac{q(s) \rho(s)}{e_{-p / a}\left(\sigma(s), t_{0}\right)}-\rho(t)[a(s) \eta(s)]^{\Delta}+\frac{\rho(s) \delta_{1}\left(s, t_{2}\right)[a(\sigma(s)) \eta(\sigma(s))]^{1+1 / \gamma}}{r(s)}\right. \\
\left.\quad-\left[\frac{r(s) \rho^{\Delta}(s)+(\gamma+1) \rho(s) \delta_{1}\left(s, t_{2}\right)[a(\sigma(s)) \eta(\sigma(s))]^{1 / \gamma}}{(\gamma+1) r^{1 /(\gamma+1)}(s) \rho^{\gamma /(\gamma+1)}(s) \delta_{1}^{\gamma /(\gamma+1)}\left(s, t_{2}\right)}\right]^{\gamma+1}\right\} \\
\quad \times \Delta s \leq \omega\left(t_{2}\right)-\omega(t) \leq \omega\left(t_{2}\right)<\infty,
\end{aligned}
$$

which contradicts (25). So the proof is complete.

In Theorem 9, if we take $\mathbb{T}$ for some special cases, then we can obtain the following corollaries.

Corollary 10. Let $\mathbb{T}=\mathbb{R}$. Assume that

$$
\int_{t_{0}}^{\infty} \frac{\left[e_{-p / a}\left(s, t_{0}\right)\right]^{1 / \gamma}}{a^{1 / \gamma}(s)} d s=\infty
$$

$$
\begin{gathered}
\int_{t_{0}}^{\infty} \frac{1}{r(s)} d s=\infty \\
\int_{t_{0}}^{\infty}\left[\frac { 1 } { r ( \xi ) } \int _ { \xi } ^ { \infty } \left(\frac{e_{-p / a}\left(\tau, t_{0}\right)}{a(\tau)}\right.\right. \\
\left.\left.\times \int_{\tau}^{\infty} \frac{q(s)}{e_{-p / a}\left(s, t_{0}\right)} d s\right)^{1 / \gamma} d \tau\right] d \xi=\infty
\end{gathered}
$$

and for all sufficiently large $T$, 


$$
\begin{aligned}
\lim _{t \rightarrow \infty} \sup \left\{\int_{T}^{t}\{\right. & L \frac{q(s) \rho(s)}{e_{-p / a}\left(s, t_{0}\right)}-\rho(t)[a(s) \eta(s)]^{\prime}+\frac{\rho(s) \delta_{1}(s, T)[a(s) \eta(s)]^{1+1 / \gamma}}{r(s)} \\
& \left.\left.-\left[\frac{r(s) \rho^{\prime}(s)+(\gamma+1) \rho(s) \delta_{1}(s, T)[a(s) \eta(\sigma(s))]^{1 / \gamma}}{(\gamma+1) r^{1 /(\gamma+1)}(s) \rho^{\gamma /(\gamma+1)}(s) \delta_{1}^{\gamma /(\gamma+1)}(s, T)}\right]^{\gamma+1}\right\} d s\right\}=\infty .
\end{aligned}
$$

Then every solution of (2) is oscillatory or tends to zero.

Corollary 11. Let $\mathbb{T}=\mathbb{Z}$ and $-p / a \in \mathfrak{R}_{+}$. Assume that

$$
\sum_{s=t_{0}}^{\infty} \frac{\left[e_{-p / a}\left(s, t_{0}\right)\right]^{1 / \gamma}}{a^{1 / \gamma}(s)}=\infty
$$

$$
\sum_{s=t_{0}}^{\infty} \frac{1}{r(s)}=\infty
$$

$$
\sum_{\xi=t_{0}}^{\infty}\left[\frac{1}{r(\xi)} \sum_{\tau=\xi}^{\infty}\left(\frac{e_{-p / a}\left(\tau, t_{0}\right)}{a(\tau)} \sum_{s=\tau}^{\infty} \frac{q(s)}{e_{-p / a}\left(s+1, t_{0}\right)}\right)^{1 / \gamma}\right]=\infty
$$

and for all sufficiently large $T$,

$$
\begin{aligned}
\lim _{t \rightarrow \infty} \sup \left\{\sum_{s=T}^{t-1}\{\right. & L \frac{q(s) \rho(s)}{e_{-p / a}\left(s+1, t_{0}\right)}-\rho(t)[a(s+1) \eta(s+1)-a(s) \eta(s)]+\frac{\rho(s) \delta_{1}(s, T)[a(s+1) \eta(s+1)]^{1+1 / \gamma}}{r(s)} \\
& \left.-\left[\frac{r(s)(\rho(s+1)-\rho(s))+(\gamma+1) \rho(s) \delta_{1}(s, T)[a(s+1) \eta(s+1)]^{1 / \gamma}}{(\gamma+1) r^{1 /(\gamma+1)}(s) \rho^{\gamma /(\gamma+1)}(s) \delta_{1}^{\gamma /(\gamma+1)}(s, T)}\right\}\right\}=\infty .
\end{aligned}
$$

Then every solution of (2) is oscillatory or tends to zero.
Theorem 12. Suppose $-p / a \in \mathfrak{R}_{+}$, and assume that (7), (8), and (13) hold, and for all sufficiently large $T$,

$$
\begin{aligned}
\lim _{t \rightarrow \infty} \sup \left\{\int _ { T } ^ { t } \left\{L \frac{q(s) \rho(s)}{e_{-p / a}\left(\sigma(s), t_{0}\right)}-\rho(s)[a(s) \eta(s)]^{\Delta}+\frac{\gamma \rho(s) \delta_{1}(s, T) \delta_{2}^{\gamma-1}(\sigma(s), T) a^{2}(\sigma(s)) \eta^{2}(\sigma(s))}{r(s)}\right.\right. \\
\left.\left.-\frac{\left[r(s) \rho^{\Delta}(s)+2 \gamma \rho(s) \delta_{1}(s, T) \delta_{2}^{\gamma-1}(\sigma(s), T) a(\sigma(s)) \eta(\sigma(s))\right]^{2}}{4 \gamma r(s) \rho(s) \delta_{1}(s, T) \delta_{2}^{\gamma-1}(\sigma(s), T)}\right\} \Delta s\right\}=\infty,
\end{aligned}
$$

where $\rho, \eta$ are defined as in Theorem 9, then every solution of (2) is oscillatory or tends to zero.

Proof. Assume (2) has a nonoscillatory solution $x$ on $\mathbb{T}_{0}$. Similar to Theorem 9 , we may assume $x(t)>0$ on $\left[t_{1}, \infty\right)_{\mathbb{T}}$, where $t_{1}$ is sufficiently large. By Lemmas 5 and 6 , there exists sufficiently large $t_{2}$ such that $\left[r(t) x^{\Delta}(t)\right]^{\Delta}>0$ on $\left[t_{2}, \infty\right)_{\mathbb{T}}$, and either $x^{\Delta}(t)>0$ on $\left[t_{2}, \infty\right)_{\mathbb{T}}$ or $\lim _{t \rightarrow \infty} x(t)=0$. Now we assume $x^{\Delta}(t)>0$ on $\left[t_{2}, \infty\right)_{\mathbb{T}}$. Let $\omega(t)$ be defined as in Theorem 9. By Lemma 7, we have the following observation:

$$
\begin{aligned}
& \frac{x^{\Delta}(t)}{x(t)} \\
& \geq \frac{\delta_{1}\left(t, t_{2}\right)}{r(t)}\left\{\frac{a^{1 / \gamma}(t)\left[r(t) x^{\Delta}(t)\right]^{\Delta}}{\left[e_{-p / a}\left(t, t_{0}\right)\right]^{1 / \gamma}}\right\} \frac{1}{x(\sigma(t))}
\end{aligned}
$$

$$
\begin{aligned}
& =\frac{\delta_{1}\left(t, t_{2}\right)}{r(t)}\left\{\frac{a^{1 / \gamma}(t)\left[r(t) x^{\Delta}(t)\right]^{\Delta}}{\left[e_{-p / a}\left(t, t_{0}\right)\right]^{1 / \gamma} x^{\gamma}(\sigma(t))}\right\} x^{\gamma-1}(\sigma(t)) \\
& \geq \frac{\delta_{1}\left(t, t_{2}\right)}{r(t)}\left\{\frac{a^{1 / \gamma}(\sigma(t))\left[r(\sigma(t)) x^{\Delta}(\sigma(t))\right]^{\Delta}}{\left[e_{-p / a}\left(\sigma(t), t_{0}\right)\right]^{1 / \gamma} x^{\gamma}(\sigma(t))}\right\} x^{\gamma-1}(\sigma(t)) \\
& \geq \frac{\delta_{1}\left(t, t_{2}\right)}{r(t)}\left\{\frac{a^{1 / \gamma}(\sigma(t))\left[r(\sigma(t)) x^{\Delta}(\sigma(t))\right]^{\Delta}}{\left[e_{-p / a}\left(\sigma(t), t_{0}\right)\right]^{1 / \gamma} x^{\gamma}(\sigma(t))}\right\} \delta_{2}^{\gamma-1} \\
& \quad \times\left(\sigma(t), t_{2}\right)\left\{\frac{a^{1 / \gamma}(\sigma(t))\left[r(\sigma(t)) x^{\Delta}(\sigma(t))\right]^{\Delta}}{\left[e_{-p / a}\left(\sigma(t), t_{0}\right)\right]^{1 / \gamma}}\right\}^{\gamma-1}
\end{aligned}
$$




$$
\begin{aligned}
& =\frac{\delta_{1}\left(t, t_{2}\right) \delta_{2}^{\gamma-1}\left(\sigma(t), t_{2}\right)}{r(t)} \\
& \times\left\{\frac{a(\sigma(t))\left(\left[r(\sigma(t)) x^{\Delta}(\sigma(t))\right]^{\Delta}\right)^{\gamma}}{e_{-p / a}\left(\sigma(t), t_{0}\right) x^{\gamma}(\sigma(t))}\right\} .
\end{aligned}
$$

$$
\begin{aligned}
& \omega^{\Delta}(t) \leq-L \frac{q(t) \rho(t)}{e_{-p / a}\left(\sigma(t), t_{0}\right)}+\frac{\rho^{\Delta}(t)}{\rho(\sigma(t))} \omega(\sigma(t))-\rho(t)\left[\frac{\gamma x^{\Delta}(t)}{x(t)}\right] \\
& \times \frac{a(\sigma(t))\left[\left(r(\sigma(t)) x^{\Delta}(\sigma(t))\right)^{\Delta}\right]^{\gamma}}{x^{\gamma}(\sigma(t)) e_{-p / a}\left(\sigma(t), t_{0}\right)}+\rho(t)[a(t) \eta(t)]^{\Delta} \\
& \leq-L \frac{q(t) \rho(t)}{e_{-p / a}\left(\sigma(t), t_{0}\right)}+\frac{\rho^{\Delta}(t)}{\rho(\sigma(t))} \omega(\sigma(t))-\gamma \rho(t) \frac{\delta_{1}\left(t, t_{2}\right) \delta_{2}^{\gamma-1}\left(\sigma(t), t_{2}\right)}{r(t)} \\
& \times\left\{\frac{a(\sigma(t))\left(\left[r(\sigma(t)) x^{\Delta}(\sigma(t))\right]^{\Delta}\right)^{\gamma}}{e_{-p / a}\left(\sigma(t), t_{0}\right) x^{\gamma}(\sigma(t))}\right\}^{2}+\rho(t)[a(t) \eta(t)]^{\Delta} \\
& =-L \frac{q(t) \rho(t)}{e_{-p / a}\left(\sigma(t), t_{0}\right)}+\frac{\rho^{\Delta}(t)}{\rho(\sigma(t))} \omega(\sigma(t))-\frac{\gamma \rho(t) \delta_{1}\left(t, t_{2}\right) \delta_{2}^{\gamma-1}\left(\sigma(t), t_{2}\right)}{r(t)} \\
& \times\left[\frac{\omega(\sigma(t))}{\rho(\sigma(t))}-a(\sigma(t)) \eta(\sigma(t))\right]^{2}+\rho(t)[a(t) \eta(t)]^{\Delta} \\
& =-L \frac{q(t) \rho(t)}{e_{-p / a}\left(\sigma(t), t_{0}\right)}+\rho(t)[a(t) \eta(t)]^{\Delta}-\frac{\gamma \rho(t) \delta_{1}\left(t, t_{2}\right) \delta_{2}^{\gamma-1}\left(\sigma(t), t_{2}\right) a^{2}(\sigma(t)) \eta^{2}(\sigma(t))}{r(t)} \\
& +\left[\frac{r(t) \rho^{\Delta}(t)+2 \gamma \rho(t) \delta_{1}\left(t, t_{2}\right) \delta_{2}^{\gamma-1}\left(\sigma(t), t_{2}\right) a(\sigma(t)) \eta(\sigma(t))}{r(t) \rho(\sigma(t))}\right] \omega(\sigma(t)) \\
& -\frac{\gamma \rho(t) \delta_{1}\left(t, t_{2}\right) \delta_{2}^{\gamma-1}\left(\sigma(t), t_{2}\right)}{r(t) \rho^{2}(\sigma(t))} \omega^{2}(\sigma(t)) \leq-L \frac{q(t) \rho(t)}{e_{-p / a}\left(\sigma(t), t_{0}\right)}+\rho(t)[a(t) \eta(t)]^{\Delta} \\
& -\frac{\gamma \rho(t) \delta_{1}\left(t, t_{2}\right) \delta_{2}^{\gamma-1}\left(\sigma(t), t_{2}\right) a^{2}(\sigma(t)) \eta^{2}(\sigma(t))}{r(t)} \\
& +\frac{\left[r(t) \rho^{\Delta}(t)+2 \gamma \rho(t) \delta_{1}\left(t, t_{2}\right) \delta_{2}^{\gamma-1}\left(\sigma(t), t_{2}\right) a(\sigma(t)) \eta(\sigma(t))\right]^{2}}{4 \gamma r(t) \rho(t) \delta_{1}\left(t, t_{2}\right) \delta_{2}^{\gamma-1}\left(\sigma(t), t_{2}\right)}
\end{aligned}
$$

Substituting $t$ with $s$ in (41), an integration for (41) with respect to $s$ from $t_{2}$ to $t$ yields

$$
\begin{aligned}
\int_{t_{2}}^{t}\{ & L \frac{q(s) \rho(s)}{e_{-p / a}\left(\sigma(s), t_{0}\right)}-\rho(s)[a(s) \eta(s)]^{\Delta}+\frac{\gamma \rho(s) \delta_{1}\left(s, t_{2}\right) \delta_{2}^{\gamma-1}\left(\sigma(s), t_{2}\right) a^{2}(\sigma(s)) \eta^{2}(\sigma(s))}{r(s)} \\
& \left.-\frac{\left[r(s) \rho^{\Delta}(s)+2 \gamma \rho(s) \delta_{1}\left(s, t_{2}\right) \delta_{2}^{\gamma-1}\left(\sigma(s), t_{2}\right) a(\sigma(s)) \eta(\sigma(s))\right]^{2}}{4 \gamma r(s) \rho(s) \delta_{1}\left(s, t_{2}\right) \delta_{2}^{\gamma-1}\left(\sigma(s), t_{2}\right)}\right\} \Delta s \leq \omega\left(t_{2}\right)-\omega(t) \leq \omega\left(t_{2}\right)<\infty
\end{aligned}
$$

which contradicts (39). So the proof is complete. 
Based on Theorems 9 and 12, we will establish some Philos-type oscillation criteria for (2).

Theorem 13. Suppose $-p / a \in \mathfrak{R}_{+}$, and assume that (7), (8), and (13) hold, and define $\mathbb{D}=\left\{(t, s) \mid t \geq s \geq t_{0}\right\}$. If there exists a function $H \in C_{\mathrm{rd}}(\mathbb{D}, \mathbb{R})$ such that

$$
\begin{gathered}
H(t, t)=0, \quad \text { for } t \geq t_{0}, \\
H(t, s)>0, \quad \text { for } t>s \geq t_{0},
\end{gathered}
$$

and $H$ has a nonpositive continuous $\Delta$ - partial derivative $H^{\Delta_{s}}(t, s)$ with respect to the second variable, and for all sufficiently large $T$,

$$
\begin{aligned}
\lim _{t \rightarrow \infty} \sup \frac{1}{H\left(t, t_{0}\right)}\left\{\int _ { t _ { 0 } } ^ { t } H ( t , s ) \left\{L \frac{q(s) \rho(s)}{e_{-p / a}\left(\sigma(s), t_{0}\right)}-\rho(t)[a(s) \eta(s)]^{\Delta}+\frac{\rho(s) \delta_{1}(s, T)[a(\sigma(s)) \eta(\sigma(s))]^{1+1 / \gamma}}{r(s)}\right.\right. \\
\left.-\left[\frac{r(s) \rho^{\Delta}(s)+(\gamma+1) \rho(s) \delta_{1}(s, T)[a(\sigma(s)) \eta(\sigma(s))]^{1 / \gamma}}{(\gamma+1) r^{1 /(\gamma+1)}(s) \rho^{\gamma /(\gamma+1)}(s) \delta_{1}^{\gamma /(\gamma+1)}(s, T)}\right\} \Delta s\right\}=\infty
\end{aligned}
$$

where $\rho, \eta$ are defined as in Theorem 9. Then every solution of (2) is oscillatory or tends to zero.

Proof. Assume (2) has a nonoscillatory solution $x$ on $\mathbb{T}_{0}$. Without loss of generality, we may assume $x(t)>0$ on $\left[t_{1}, \infty\right)_{\mathbb{V}}$, where $t_{1}$ is sufficiently large. By Lemmas 5 and 6 , there exists sufficiently large $t_{2}$ such that $\left[r(t) x^{\Delta}(t)\right]^{\Delta}>0$ on $\left[t_{2}, \infty\right)_{\mathbb{T}}$, and either $x^{\Delta}(t)>0$ on $\left[t_{2}, \infty\right)_{\mathbb{T}}$ or $\lim _{t \rightarrow \infty} x(t)=$ 0 . Now we assume $x^{\Delta}(t)>0$ on $\left[t_{2}, \infty\right)_{\mathbb{T}}$. Let $\omega(t)$ be defined as in Theorem 9. By (33) we have

$$
\begin{aligned}
& +\frac{\rho(t) \delta_{1}\left(t, t_{2}\right)[a(\sigma(t)) \eta(\sigma(t))]^{1+1 / \gamma}}{r(t)} \\
& -\left[\frac{r(t) \rho^{\Delta}(t)+(\gamma+1) \rho(t) \delta_{1}\left(t, t_{2}\right)[a(\sigma(t)) \eta(\sigma(t))]^{1 / \gamma}}{(\gamma+1) r^{1 /(\gamma+1)}(t) \rho^{\gamma /(\gamma+1)}(t) \delta_{1}^{\gamma /(\gamma+1)}\left(t, t_{2}\right)}\right]^{\gamma+1} \\
& \leq-\omega^{\Delta}(t) .
\end{aligned}
$$

$$
L \frac{q(t) \rho(t)}{e_{-p / a}\left(\sigma(t), t_{0}\right)}-\rho(t)[a(t) \eta(t)]^{\Delta}
$$

Substituting $t$ with $s$ in (45) and multiplying both sides by $H(t, s)$ and then integrating with respect to $s$ from $t_{2}$ to $t$ yield

$$
\begin{gathered}
\int_{t_{2}}^{t} H(t, s)\left\{L \frac{q(s) \rho(s)}{e_{-p / a}\left(\sigma(s), t_{0}\right)}-\rho(t)[a(s) \eta(s)]^{\Delta}+\frac{\rho(s) \delta_{1}\left(s, t_{2}\right)[a(\sigma(s)) \eta(\sigma(s))]^{1+1 / \gamma}}{r(s)}\right. \\
\quad-\left[\frac{r(s) \rho^{\Delta}(s)+(\gamma+1) \rho(s) \delta_{1}\left(s, t_{2}\right)[a(\sigma(s)) \eta(\sigma(s))]^{1 / \gamma}}{(\gamma+1) r^{1 /(\gamma+1)}(s) \rho^{\gamma /(\gamma+1)}(s) \delta_{1}^{\gamma /(\gamma+1)}\left(s, t_{2}\right)}\right] \Delta s \\
\leq-\int_{t_{2}}^{t} H(t, s) \omega^{\Delta}(s) \Delta s=H\left(t, t_{2}\right) \omega\left(t_{2}\right)+\int_{t_{2}}^{t} H^{\Delta_{s}}(t, s) \omega(\sigma(s)) \Delta s \leq H\left(t, t_{2}\right) \omega\left(t_{2}\right) \leq H\left(t, t_{0}\right) \omega\left(t_{2}\right) .
\end{gathered}
$$

Then

$$
\begin{gathered}
\int_{t_{0}}^{t} H(t, s)\left\{L \frac{q(s) \rho(s)}{e_{-p / a}\left(\sigma(s), t_{0}\right)}-\rho(t)[a(s) \eta(s)]^{\Delta}+\frac{\rho(s) \delta_{1}\left(s, t_{2}\right)[a(\sigma(s)) \eta(\sigma(s))]^{1+1 / \gamma}}{r(s)}\right. \\
-\left[\frac{r(s) \rho^{\Delta}(s)+(\gamma+1) \rho(s) \delta_{1}\left(s, t_{2}\right)[a(\sigma(s)) \eta(\sigma(s))]^{1 / \gamma}}{(\gamma+1) r^{1 /(\gamma+1)}(s) \rho^{\gamma /(\gamma+1)}(s) \delta_{1}^{\gamma /(\gamma+1)}\left(s, t_{2}\right)}\right\} \Delta s \\
=\int_{t_{0}}^{t_{2}} H(t, s)\left\{L \frac{q(s) \rho(s)}{e_{-p / a}\left(\sigma(s), t_{0}\right)}-\rho(t)[a(s) \eta(s)]^{\Delta}+\frac{\rho(s) \delta_{1}\left(s, t_{2}\right)[a(\sigma(s)) \eta(\sigma(s))]^{1+1 / \gamma}}{r(s)}\right. \\
\left.-\left[\frac{r(s) \rho^{\Delta}(s)+(\gamma+1) \rho(s) \delta_{1}\left(s, t_{2}\right)[a(\sigma(s)) \eta(\sigma(s))]^{1 / \gamma}}{(\gamma+1) r^{1 /(\gamma+1)}(s) \rho^{\gamma /(\gamma+1)}(s) \delta_{1}^{\gamma /(\gamma+1)}\left(s, t_{2}\right)}\right]_{\Delta s}^{\gamma+1}\right\}
\end{gathered}
$$




$$
\begin{aligned}
& +\int_{t_{2}}^{t} H(t, s)\left\{L \frac{q(s) \rho(s)}{e_{-p / a}\left(\sigma(s), t_{0}\right)}-\rho(t)[a(s) \eta(s)]^{\Delta}+\frac{\rho(s) \delta_{1}\left(s, t_{2}\right)[a(\sigma(s)) \eta(\sigma(s))]^{1+1 / \gamma}}{r(s)}\right. \\
& \left.\quad-\left[\frac{r(s) \rho^{\Delta}(s)+(\gamma+1) \rho(s) \delta_{1}\left(s, t_{2}\right)[a(\sigma(s)) \eta(\sigma(s))]^{1 / \gamma}}{(\gamma+1) r^{1 /(\gamma+1)}(s) \rho^{\gamma /(\gamma+1)}(s) \delta_{1}^{\gamma /(\gamma+1)}\left(s, t_{2}\right)}\right]^{\gamma+1}\right\} \Delta s \\
& \leq H\left(t, t_{0}\right) \omega\left(t_{2}\right)+H\left(t, t_{0}\right) \\
& \times \int_{t_{0}}^{t_{2}} \mid L \frac{q(s) \rho(s)}{e_{-p / a}\left(\sigma(s), t_{0}\right)}-\rho(t)[a(s) \eta(s)]^{\Delta}+\frac{\rho(s) \delta_{1}\left(s, t_{2}\right)[a(\sigma(s)) \eta(\sigma(s))]^{1+1 / \gamma}}{r(s)} \\
& -\left[\frac{r(s) \rho^{\Delta}(s)+(\gamma+1) \rho(s) \delta_{1}\left(s, t_{2}\right)[a(\sigma(s)) \eta(\sigma(s))]^{1 / \gamma}}{(\gamma+1) r^{1 /(\gamma+1)}(s) \rho^{\gamma /(\gamma+1)}(s) \delta_{1}^{\gamma /(\gamma+1)}\left(s, t_{2}\right)}\right]^{\gamma+1} \mid \Delta s .
\end{aligned}
$$

So

$$
\begin{gathered}
\lim _{t \rightarrow \infty} \sup \frac{1}{H\left(t, t_{0}\right)}\left\{\int _ { t _ { 0 } } ^ { t } H ( t , s ) \left\{L \frac{q(s) \rho(s)}{e_{-p / a}\left(\sigma(s), t_{0}\right)}-\rho(t)[a(s) \eta(s)]^{\Delta}+\frac{\rho(s) \delta_{1}\left(s, t_{2}\right)[a(\sigma(s)) \eta(\sigma(s))]^{1+1 / \gamma}}{r(s)}\right.\right. \\
\left.\quad-\left[\frac{r(s) \rho^{\Delta}(s)+(\gamma+1) \rho(s) \delta_{1}\left(s, t_{2}\right)[a(\sigma(s)) \eta(\sigma(s))]^{1 / \gamma}}{(\gamma+1) r^{1 /(\gamma+1)}(s) \rho^{\gamma /(\gamma+1)}(s) \delta_{1}^{\gamma /(\gamma+1)}\left(s, t_{2}\right)}\right\} \Delta s\right\} \\
\leq \omega\left(t_{2}\right)+\int_{t_{0}}^{t_{2}} \mid L \frac{q(s) \rho(s)}{e_{-p / a}\left(\sigma(s), t_{0}\right)}-\rho(t)[a(s) \eta(s)]^{\Delta}+\frac{\rho(s) \delta_{1}\left(s, t_{2}\right)[a(\sigma(s)) \eta(\sigma(s))]^{1+1 / \gamma}}{r(s)} \\
-\left[\frac{r(s) \rho^{\Delta}(s)+(\gamma+1) \rho(s) \delta_{1}\left(s, t_{2}\right)[a(\sigma(s)) \eta(\sigma(s))]^{1 / \gamma}}{(\gamma+1) r^{1 /(\gamma+1)}(s) \rho^{\gamma /(\gamma+1)}(s) \delta_{1}^{\gamma /(\gamma+1)}\left(s, t_{2}\right)}\right]^{\gamma+1} \mid \Delta s<\infty,
\end{gathered}
$$

which contradicts (44). So the proof is complete.

Theorem 14. Suppose $-p / a \in \mathfrak{R}_{+}$, and assume that (7), (8), and (13) hold. Let $H$ be defined as in Theorem 13, and for all sufficiently large $T$,

$$
\begin{aligned}
\lim _{t \rightarrow \infty} \sup \frac{1}{H\left(t, t_{0}\right)}\left\{\int_{t_{0}}^{t} H(t, s)\{\right. & L \frac{q(s) \rho(s)}{e_{-p / a}\left(\sigma(s), t_{0}\right)}-\rho(s)[a(s) \eta(s)]^{\Delta} \\
& +\frac{\gamma \rho(s) \delta_{1}(s, T) \delta_{2}^{\gamma-1}(\sigma(s), T) a^{2}(\sigma(s)) \eta^{2}(\sigma(s))}{r(s)} \\
& \left.\left.-\frac{\left[r(s) \rho^{\Delta}(s)+2 \gamma \rho(s) \delta_{1}(s, T) \delta_{2}^{\gamma-1}(\sigma(s), T) a(\sigma(s)) \eta(\sigma(s))\right]^{2}}{4 \gamma r(s) \rho(s) \delta_{1}(s, T) \delta_{2}^{\gamma-1}(\sigma(s), T)}\right\} \Delta s\right\}=\infty,
\end{aligned}
$$

where $\rho, \eta$ are defined as in Theorem 9. Then every solution of (2) is oscillatory or tends to zero.

Proof. Assume (2) has a nonoscillatory solution $x$ on $\mathbb{T}_{0}$. Without loss of generality, we may assume $x(t)>0$ on $\left[t_{1}, \infty\right)_{\mathbb{T}}$, where $t_{1}$ is sufficiently large. By Lemmas 5 and 6, there exists sufficiently large $t_{2}$ such that $\left[r(t) x^{\Delta}(t)\right]^{\Delta}>$ 0 on $\left[t_{2}, \infty\right)_{\mathbb{T}}$, and either $x^{\Delta}(t)>0$ on $\left[t_{2}, \infty\right)_{\mathbb{T}}$ or $\lim _{t \rightarrow \infty} x(t)=0$. Now we assume $x^{\Delta}(t)>0$ on $\left[t_{2}, \infty\right)_{\mathbb{T}}$. Let $\omega(t)$ be defined as in Theorem 9. By (41) we have 


$$
\begin{gathered}
L \frac{q(t) \rho(t)}{e_{-p / a}\left(\sigma(t), t_{0}\right)}-\rho(t)[a(t) \eta(t)]^{\Delta}+\frac{\gamma \rho(t) \delta_{1}\left(t, t_{2}\right) \delta_{2}^{\gamma-1}\left(\sigma(t), t_{2}\right) a^{2}(\sigma(t)) \eta^{2}(\sigma(t))}{r(t)} \\
-\frac{\left[r(t) \rho^{\Delta}(t)+2 \gamma \rho(t) \delta_{1}\left(t, t_{2}\right) \delta_{2}^{\gamma-1}\left(\sigma(t), t_{2}\right) a(\sigma(t)) \eta(\sigma(t))\right]^{2}}{4 \gamma r(t) \rho(t) \delta_{1}\left(t, t_{2}\right) \delta_{2}^{\gamma-1}\left(\sigma(t), t_{2}\right)} \leq-\omega^{\Delta}(t) .
\end{gathered}
$$

Substituting $t$ with $s$ in (45), multiplying both sides by $H(t, s)$, and then integrating with respect to $s$ from $t_{2}$ to $t$ yield

$$
\begin{aligned}
& \int_{t_{2}}^{t} H(t, s)\left\{L \frac{q(s) \rho(s)}{e_{-p / a}\left(\sigma(s), t_{0}\right)}-\rho(s)[a(s) \eta(s)]^{\Delta}\right. \\
&+\frac{\gamma \rho(s) \delta_{1}\left(s, t_{2}\right) \delta_{2}^{\gamma-1}\left(\sigma(s), t_{2}\right) a^{2}(\sigma(s)) \eta^{2}(\sigma(s))}{r(s)} \\
&\left.-\frac{\left[r(s) \rho^{\Delta}(s)+2 \gamma \rho(s) \delta_{1}\left(s, t_{2}\right) \delta_{2}^{\gamma-1}\left(\sigma(s), t_{2}\right) a(\sigma(s)) \eta(\sigma(s))\right]^{2}}{4 \gamma r(s) \rho(s) \delta_{1}\left(s, t_{2}\right) \delta_{2}^{\gamma-1}\left(\sigma(s), t_{2}\right)}\right\} \Delta s \\
& \leq-\int_{t_{2}}^{t} H(t, s) \omega^{\Delta}(s) \Delta s=H\left(t, t_{2}\right) \omega\left(t_{2}\right)+\int_{t_{2}}^{t} H^{\Delta_{s}}(t, s) \omega(\sigma(s)) \Delta s \\
& \leq H\left(t, t_{2}\right) \omega\left(t_{2}\right) \leq H\left(t, t_{0}\right) \omega\left(t_{2}\right) .
\end{aligned}
$$

Then similar to Theorem 13, we obtain

$$
\begin{aligned}
& \lim _{t \rightarrow \infty} \sup \frac{1}{H\left(t, t_{0}\right)} \\
& \times\left\{\int _ { t _ { 0 } } ^ { t } H ( t , s ) \left\{L \frac{q(s) \rho(s)}{e_{-p / a}\left(\sigma(s), t_{0}\right)}-\rho(s)[a(s) \eta(s)]^{\Delta}+\frac{\gamma \rho(s) \delta_{1}\left(s, t_{2}\right) \delta_{2}^{\gamma-1}\left(\sigma(s), t_{2}\right) a^{2}(\sigma(s)) \eta^{2}(\sigma(s))}{r(s)}\right.\right. \\
& \left.\left.-\frac{\left[r(s) \rho^{\Delta}(s)+2 \gamma \rho(s) \delta_{1}\left(s, t_{2}\right) \delta_{2}^{\gamma-1}\left(\sigma(s), t_{2}\right) a(\sigma(s)) \eta(\sigma(s))\right]^{2}}{4 \gamma r(s) \rho(s) \delta_{1}\left(s, t_{2}\right) \delta_{2}^{\gamma-1}\left(\sigma(s), t_{2}\right)}\right\} \Delta s\right\} \\
& \leq \omega\left(t_{2}\right)+\int_{t_{0}}^{t_{2}} \mid L \frac{q(s) \rho(s)}{e_{-p / a}\left(\sigma(s), t_{0}\right)}-\rho(s)[a(s) \eta(s)]^{\Delta} \\
& +\frac{\gamma \rho(s) \delta_{1}\left(s, t_{2}\right) \delta_{2}^{\gamma-1}\left(\sigma(s), t_{2}\right) a^{2}(\sigma(s)) \eta^{2}(\sigma(s))}{r(s)} \\
& -\frac{\left[r(s) \rho^{\Delta}(s)+2 \gamma \rho(s) \delta_{1}\left(s, t_{2}\right) \delta_{2}^{\gamma-1}\left(\sigma(s), t_{2}\right) a(\sigma(s)) \eta(\sigma(s))\right]^{2}}{4 \gamma r(s) \rho(s) \delta_{1}\left(s, t_{2}\right) \delta_{2}^{\gamma-1}\left(\sigma(s), t_{2}\right)} \mid \Delta s<\infty,
\end{aligned}
$$

which contradicts (44). So the proof is complete. 
In Theorems 13 and 14 , if we take $H(t, s)$ for some special functions such as $(t-s)^{m}$ or $\ln (t / s)$, then we can obtain some corollaries. For example, if we take $H(t, s)=(t-s)^{m}, m \geq 1$, then we have the following corollaries.

Corollary 15. Suppose $-p / a \in \mathfrak{R}_{+}$, and assume that (7), (8), and (13) hold, and for all sufficiently large $T$,

$$
\begin{aligned}
& \lim _{t \rightarrow \infty} \sup \frac{1}{\left(t-t_{0}\right)^{m}} \\
& \times\left\{\int _ { t _ { 0 } } ^ { t } ( t - s ) ^ { m } \left\{L \frac{q(s) \rho(s)}{e_{-p / a}\left(\sigma(s), t_{0}\right)}-\rho(t)[a(s) \eta(s)]^{\Delta}+\frac{\rho(s) \delta_{1}(s, T)[a(\sigma(s)) \eta(\sigma(s))]^{1+1 / \gamma}}{r(s)}\right.\right. \\
& \left.\left.-\left[\frac{r(s) \rho^{\Delta}(s)+(\gamma+1) \rho(s) \delta_{1}(s, T)[a(\sigma(s)) \eta(\sigma(s))]^{1 / \gamma}}{(\gamma+1) r^{1 /(\gamma+1)}(s) \rho^{\gamma /(\gamma+1)}(s) \delta_{1}^{\gamma /(\gamma+1)}(s, T)}\right]^{\gamma+1}\right\} \Delta s\right\}=\infty .
\end{aligned}
$$

Then every solution of (2) is oscillatory or tends to zero.

Corollary 16. Suppose $-p / a \in \mathfrak{R}_{+}$, and assume that (7), (8), and (13) hold, and for all sufficiently large $T$,

$$
\begin{aligned}
& \lim _{t \rightarrow \infty} \sup \frac{1}{\left(t-t_{0}\right)^{m}} \\
& \times\left\{\int _ { t _ { 0 } } ^ { t } ( t - s ) ^ { m } \left\{L \frac{q(s) \rho(s)}{e_{-p / a}\left(\sigma(s), t_{0}\right)}-\rho(s)[a(s) \eta(s)]^{\Delta}+\frac{\gamma \rho(s) \delta_{1}(s, T) \delta_{2}^{\gamma-1}(\sigma(s), T) a^{2}(\sigma(s)) \eta^{2}(\sigma(s))}{r(s)}\right.\right. \\
& \\
&\left.\left.\quad-\frac{\left[r(s) \rho^{\Delta}(s)+2 \gamma \rho(s) \delta_{1}(s, T) \delta_{2}^{\gamma-1}(\sigma(s), T) a(\sigma(s)) \eta(\sigma(s))\right]^{2}}{4 \gamma r(s) \rho(s) \delta_{1}(s, T) \delta_{2}^{\gamma-1}(\sigma(s), T)}\right\} \Delta s\right\}=\infty .
\end{aligned}
$$

Then every solution of (2) is oscillatory or tends to zero.

Remark 17. The established results above extend the main results in [25, Theorems 2.1-2.4] except that the latter is related to time delay.

Remark 18. In Theorems $12-14$, if we take $\mathbb{T}$ for some special time scales, we can obtain similar results as in Corollaries 10 and 11, which are omitted here.

In Theorems $9,12,13$, and 14 , if we let $p=0$, then $e_{-p / a}\left(t, t_{0}\right) \equiv 1$, and subsequently we obtain the following four corollaries concerning oscillatory criteria of the following equation:

$$
\left(a(t)\left(\left[r(t) x^{\Delta}(t)\right]^{\Delta}\right)^{\gamma}\right)^{\Delta}+q(t) f(x(t))=0, \quad t \in \mathbb{T}_{0} .
$$

Corollary 19. Assume (8) holds. If

$$
\int_{t_{0}}^{\infty} \frac{1}{a^{1 / \gamma}(s)} \Delta s=\infty,
$$

$\lim _{t \rightarrow \infty} \sup \int_{t_{0}}^{t}\left[\frac{1}{r(\xi)} \int_{\xi}^{\infty}\left(\frac{1}{a(\tau)} \int_{\tau}^{\infty} q(s) \Delta s\right)^{1 / \gamma} \Delta \tau\right] \Delta \xi=\infty$.

and for all sufficiently large $T$,

$$
\begin{aligned}
\lim _{t \rightarrow \infty} \sup \left\{\int _ { T } ^ { t } \left\{L q(s) \rho(s)-\rho(t)[a(s) \eta(s)]^{\Delta}+\frac{\rho(s) \delta_{1}(s, T)[a(\sigma(s)) \eta(\sigma(s))]^{1+1 / \gamma}}{r(s)}\right.\right. \\
\left.\left.\quad-\left[\frac{r(s) \rho^{\Delta}(s)+(\gamma+1) \rho(s) \delta_{1}(s, T)[a(\sigma(s)) \eta(\sigma(s))]^{1 / \gamma}}{(\gamma+1) r^{1 /(\gamma+1)}(s) \rho^{\gamma /(\gamma+1)}(s) \delta_{1}^{\gamma /(\gamma+1)}(s, T)}\right]^{\gamma+1}\right\} \Delta s\right\}=\infty
\end{aligned}
$$


where $\rho, \eta$ are defined as in Theorem 9. Then every solution of (2) is oscillatory or tends to zero.
Corollary 20. Assume that (8), (56), and (57) hold, and for all sufficiently large $T$,

$$
\begin{aligned}
\lim _{t \rightarrow \infty} \sup \left\{\int _ { T } ^ { t } \left\{L q(s) \rho(s)-\rho(s)[a(s) \eta(s)]^{\Delta}+\frac{\gamma \rho(s) \delta_{1}(s, T) \delta_{2}^{\gamma-1}(\sigma(s), T) a^{2}(\sigma(s)) \eta^{2}(\sigma(s))}{r(s)}\right.\right. \\
\left.\left.-\frac{\left[r(s) \rho^{\Delta}(s)+2 \gamma \rho(s) \delta_{1}(s, T) \delta_{2}^{\gamma-1}(\sigma(s), T) a(\sigma(s)) \eta(\sigma(s))\right]^{2}}{4 \gamma r(s) \rho(s) \delta_{1}(s, T) \delta_{2}^{\gamma-1}(\sigma(s), T)}\right\} \Delta s\right\}=\infty,
\end{aligned}
$$

where $\rho$, $\eta$ are defined as in Theorem 9. Then every solution of (2) is oscillatory or tends to zero.
Corollary 21. Assume that (8), (56), and (57) hold. If for all sufficiently large $T$,

$$
\begin{aligned}
\lim _{t \rightarrow \infty} \sup \frac{1}{H\left(t, t_{0}\right)}\left\{\int _ { t _ { 0 } } ^ { t } H ( t , s ) \left\{L q(s) \rho(s)-\rho(t)[a(s) \eta(s)]^{\Delta}+\frac{\rho(s) \delta_{1}(s, T)[a(\sigma(s)) \eta(\sigma(s))]^{1+1 / \gamma}}{r(s)}\right.\right. \\
\left.-\left[\frac{r(s) \rho^{\Delta}(s)+(\gamma+1) \rho(s) \delta_{1}(s, T)[a(\sigma(s)) \eta(\sigma(s))]^{1 / \gamma}}{(\gamma+1) r^{1 /(\gamma+1)}(s) \rho^{\gamma /(\gamma+1)}(s) \delta_{1}^{\gamma /(\gamma+1)}(s, T)}\right\} \Delta s\right\}=\infty
\end{aligned}
$$

where $\rho, \eta, H$ are defined as in Theorems 9 and 13, respectively, then every solution of (2) is oscillatory or tends to zero.
Corollary 22. Assume that (8), (56), and (57) hold. If for all sufficiently large $T$,

$$
\begin{aligned}
\lim _{t \rightarrow \infty} \sup \frac{1}{H\left(t, t_{0}\right)}\left\{\int_{t_{0}}^{t} H(t, s)\{\right. & \frac{q(s) \rho(s)}{e_{-p / a}\left(\sigma(s), t_{0}\right)}-\rho(s)[a(s) \eta(s)]^{\Delta}+\frac{\gamma \rho(s) \delta_{1}(s, T) \delta_{2}^{\gamma-1}(\sigma(s), T) a^{2}(\sigma(s)) \eta^{2}(\sigma(s))}{r(s)} \\
& \left.\left.-\frac{\left[r(s) \rho^{\Delta}(s)+2 \gamma \rho(s) \delta_{1}(s, T) \delta_{2}^{\gamma-1}(\sigma(s), T) a(\sigma(s)) \eta(\sigma(s))\right]^{2}}{4 \gamma r(s) \rho(s) \delta_{1}(s, T) \delta_{2}^{\gamma-1}(\sigma(s), T)}\right\} \Delta s\right\}=\infty,
\end{aligned}
$$

where $\rho, \eta, H$ are defined as in Theorems 9 and 13, respectively, then every solution of (2) is oscillatory or tends to zero.

Remark 23. In [34, Theorems 3.3-3.4] and [35, Theorems 2.7-2.9], Saker established some new oscillatory criteria for the equation

$$
\left(p(t)\left[\left(r(t) x^{\Delta}(t)\right)^{\Delta}\right]^{\gamma}\right)^{\Delta}+q(t) f(x(\tau(t)))=0
$$

under the condition $\tau(t) \leq t$. We note that the conditions (8) and (56) in Corollaries 19-22 are consistent with those in $[34,(24)]$ and $[35,(1.12)]$, which were used in $[34$, Theorems 3.3-3.4] and [35, Theorems 2.7-2.9], respectively, while $r(t) \equiv 1$ is assumed in $[34,35]$. Moreover, in the results established above, the Riccati substitution function is defined by $\omega(t)=\rho(t) a(t)\left[\left(\left[r(t) x^{\Delta}(t)\right]^{\Delta}\right)^{\gamma} / x^{\gamma}(t) e_{-p / a}\left(t, t_{0}\right)+\eta(t)\right]$ (see Theorem 9), which is different form that in [34, Theorem 3.3] and [35, Theorem 2.7], where the Riccati substitution function is defined by $u(t)=y^{[2]} /\left(y^{[1]}\right)^{\gamma}=x^{\Delta \Delta} /\left(x^{\Delta}\right)^{\gamma}$. Since the Riccati substitution function is the most important fact in establishing sufficient oscillatory conditions, so our results in Corollaries 19-22 are essentially different from Saker's results in $[34,35]$.

\section{Applications}

In this section, we will present some applications for the established results above. First we consider the following third-order nonlinear differential equation with damping term.

Example 24. Consider

$$
\left[\left(t x^{\prime \prime}(t)\right)^{\gamma}\right]^{\prime}+\frac{1}{t^{\gamma+1}}\left(x^{\prime \prime}(t)\right)^{\gamma}
$$




$$
+\frac{1}{t^{\gamma+1}} x^{\gamma}(t)\left[e^{x(t)}+1\right]=0, \quad t \in[2, \infty)
$$

where $\gamma \geq 1$ is a quotient of two odd positive integers.

We have in $(2) \mathbb{T}=\mathbb{R}, a(t)=t^{\gamma}, p(t)=q(t)=1 / t^{\gamma+1}$, $f(x)=x^{\gamma}\left[e^{x}+1\right], r(t)=1, t_{0}=2$. Then $f(x) / x^{\gamma} \geq 1=$ $L, \mu(t)=\sigma(t)-t=0$, and $-p / a \in \mathfrak{R}_{+}$. So $e_{-p / a}\left(t, t_{0}\right)=$ $e_{-p / a}(t, 2)=\exp \left(-\int_{2}^{t}(p(s) / a(s)) d s\right)$. Moreover, we have

$$
\begin{aligned}
1 & >\exp \left(-\int_{2}^{t} \frac{p(s)}{a(s)} d s\right) \geq 1-\int_{2}^{t} \frac{p(s)}{a(s)} d s \\
& =1-\int_{2}^{t} \frac{1}{s^{2 \gamma+1}} d s=1+\frac{1}{2 \gamma}\left[t^{-2 \gamma}-2^{-2 \gamma}\right]>\frac{1}{2} .
\end{aligned}
$$

Then we have

$$
\begin{gathered}
\int_{t_{0}}^{\infty} \frac{\left[e_{-p / a}\left(s, t_{0}\right)\right]^{1 / \gamma}}{a^{1 / \gamma}(s)} d s>\frac{1}{2^{1 / \gamma}} \int_{2}^{\infty} \frac{1}{s} d s=\infty \\
\int_{t_{0}}^{\infty} \frac{1}{r(s)} d s=\infty
\end{gathered}
$$

Furthermore,

$$
\begin{aligned}
& \int_{t_{0}}^{\infty}\left[\frac{1}{r(\xi)} \int_{\xi}^{\infty}\left(\frac{e_{-p / a}\left(\tau, t_{0}\right)}{a(\tau)} \int_{\tau}^{\infty} \frac{q(s)}{e_{-p / a}\left(s, t_{0}\right)} d s\right)^{1 / \gamma} d \tau\right] d \xi \\
& =\int_{2}^{\infty}\left[\int_{\xi}^{\infty}\left(\frac{e_{-p / a}(\tau, 2)}{\tau^{\gamma}} \int_{\tau}^{\infty} \frac{1}{s^{\gamma+1} e_{-p / a}(s, 2)} d s\right)^{1 / \gamma} d \tau\right] d \xi \\
& >\frac{1}{2^{1 / \gamma}} \int_{2}^{\infty}\left[\int_{\xi}^{\infty}\left(\frac{1}{\tau^{\gamma}} \int_{\tau}^{\infty} \frac{1}{s^{\gamma+1}} d s\right)^{1 / \gamma} d \tau\right] d \xi \\
& =\frac{1}{(2 \gamma)^{1 / \gamma}} \int_{2}^{\infty}\left[\int_{\xi}^{\infty} \frac{1}{\tau^{2}} d \tau\right] d \xi=\frac{1}{(2 \gamma)^{1 / \gamma}} \int_{2}^{\infty} \frac{1}{\xi} d \xi=\infty
\end{aligned}
$$

On the other hand, for a sufficiently large $T$, we have

$$
\delta_{1}(t, T)=\int_{T}^{t} \frac{\left[e_{-p / a}\left(s, t_{0}\right)\right]^{1 / \gamma}}{a^{1 / \gamma}(s)} d s>\frac{1}{2^{1 / \gamma}} \int_{T}^{t} \frac{1}{s} d s \longrightarrow \infty .
$$

(65) So there exists a sufficiently large $T^{*}>T$ such that $\delta_{1}(t, T)>$ 1 for $t \in\left[T^{*}, \infty\right)$. Taking $\rho(t)=t^{\gamma}, \eta(t)=0$ in (36), we get that

$$
\begin{aligned}
& \lim _{t \rightarrow \infty} \sup \left\{\int_{T}^{t}\left\{L \frac{q(s) \rho(s)}{e_{-p / a}\left(s, t_{0}\right)}-\left[\frac{r(s) \rho^{\prime}(s)}{(\gamma+1) r^{1 /(\gamma+1)}(s) \rho^{\gamma /(\gamma+1)}(s) \delta_{1}^{\gamma /(\gamma+1)}(s, T)}\right]^{\gamma+1}\right\} d s\right\}\left\{\int_{T}^{T^{*}}\left\{L \frac{q(s) \rho(s)}{e_{-p / a}\left(s, t_{0}\right)}-\left[\frac{r(s) \rho^{\prime}(s)}{(\gamma+1) r^{1 /(\gamma+1)}(s) \rho^{\gamma /(\gamma+1)}(s) \delta_{1}^{\gamma /(\gamma+1)}(s, T)}\right]^{\gamma+1}\right\} d s\right. \\
&=\lim _{t \rightarrow \infty} \sup \{\left.+\int_{T^{*}}^{t}\left\{L \frac{q(s) \rho(s)}{e_{-p / a}\left(s, t_{0}\right)}-\left[\frac{r(s) \rho^{\prime}(s)}{(\gamma+1) r^{1 /(\gamma+1)}(s) \rho^{\gamma /(\gamma+1)}(s) \delta_{1}^{\gamma /(\gamma+1)}(s, T)}\right]^{\gamma+1}\right\} d s\right\} \\
&>\lim _{t \rightarrow \infty} \sup \left\{\int_{T}^{T^{*}}\left\{L \frac{q(s) \rho(s)}{e_{-p / a}\left(s, t_{0}\right)}-\left[\frac{r(s) \rho^{\prime}(s)}{(\gamma+1) r^{1 /(\gamma+1)}(s) \rho^{\gamma /(\gamma+1)}(s) \delta_{1}^{\gamma /(\gamma+1)}(s, T)}\right]^{\gamma+1}\right\} d s\right. \\
&\left.+\int_{T^{*}}^{t}\left[1-\left(\frac{\gamma}{\gamma+1}\right)^{\gamma+1}\right] \frac{1}{s} d s\right\} \longrightarrow \infty .
\end{aligned}
$$

So (35)-(36) all hold, and by Corollary 10 we deduce that every solution of (63) is oscillatory or tends to zero.

Next we consider the following third-order difference equation:

\section{Example 25. Consider}

$$
\begin{array}{r}
\Delta\left[\left(t \Delta^{2} x(t)\right)^{\gamma}\right]+\frac{1}{t^{\gamma+1}}\left(\Delta^{2} x(t)\right)^{\gamma}+\frac{M}{t^{\gamma+1}} x^{\gamma}(t)=0, \\
t \in[2, \infty)_{\mathbb{Z}},
\end{array}
$$

where $\Delta$ denotes the difference operator, $M>0$ is a constant, and $\gamma \geq 1$ is a quotient of two odd positive integers.

We have in $(2) \mathbb{T}=\mathbb{Z}, a(t)=t^{\gamma}, p(t)=q(t)=1 / t^{\gamma+1}$, $f(x)=M x^{\gamma}, r(t)=1, t_{0}=2$. Then $f(x) / x^{\gamma} \geq M=$ $L, \mu(t)=\sigma(t)-t=1$, and

$$
1-\mu(t) \frac{p(t)}{a(t)}=1-\frac{1}{t^{2 \gamma+1}} \geq 1-\frac{1}{2}>0,
$$

which implies $-p / a \in \mathfrak{R}_{+}$. So by [2, Lemma 2] we obtain

$$
e_{-p / a}\left(t, t_{0}\right)=e_{-p / a}(t, 2) \geq 1-\int_{2}^{t} \frac{p(s)}{a(s)} \Delta s
$$




$$
\begin{gathered}
=1-\int_{2}^{t} \frac{1}{s^{2 \gamma+1}} \Delta s=1-\sum_{s=2}^{t-1} \frac{1}{s^{2 \gamma+1}} \\
\geq 1-\int_{1}^{t-1} \frac{1}{s^{2 \gamma+1}} d s \\
=1+\frac{1}{2 \gamma}\left[(t-1)^{-2 \gamma}-1\right]>\frac{1}{2} \\
e_{-p / a}\left(t, t_{0}\right) \leq \exp \left(-\int_{2}^{t} \frac{p(s)}{a(s)} \Delta s\right)<1 .
\end{gathered}
$$

Then we have

$$
\begin{aligned}
& \sum_{s=t_{0}}^{\infty} \frac{\left[e_{-p / a}\left(s, t_{0}\right)\right]^{1 / \gamma}}{a^{1 / \gamma}(s)} \\
& =\sum_{s=2}^{\infty} \frac{\left[e_{-p / a}(s, 2)\right]^{1 / \gamma}}{a^{1 / \gamma}(s)} \\
& =\sum_{s=2}^{\infty} \frac{\left[e_{-p / a}(s, 2)\right]^{1 / \gamma}}{s}>\frac{1}{2^{1 / \gamma}} \sum_{s=2}^{\infty} \frac{1}{s}=\infty, \\
& \sum_{s=t_{0}}^{\infty} \frac{1}{r(s)}=\infty .
\end{aligned}
$$

Furthermore,

$$
\sum_{\xi=t_{0}}^{\infty}\left[\frac{1}{r(\xi)} \sum_{\tau=\xi}^{\infty}\left(\frac{e_{-p / a}\left(\tau, t_{0}\right)}{a(\tau)} \sum_{s=\tau}^{\infty} \frac{q(s)}{e_{-p / a}\left(s+1, t_{0}\right)}\right)^{1 / \gamma}\right]
$$

$$
\begin{aligned}
& =\sum_{\xi=2}^{\infty}\left[\frac { 1 } { r ( \xi ) } \sum _ { \tau = \xi } ^ { \infty } \left(\frac{e_{-p / a}(\tau, 2)}{a(\tau)}\right.\right. \\
& >\frac{1}{2^{1 / \gamma}} \sum_{\xi=2}^{\infty}\left[\sum_{\tau=\tau}^{\infty}\left(\frac{1}{\tau^{\gamma}} \sum_{s=\tau}^{\infty} \frac{1}{s^{\gamma / a}}\right)^{1 / \gamma}\right] \\
& \left.s^{1 / \gamma}\right] \\
& >\frac{1}{2^{1 / \gamma}} \sum_{\xi=2}^{\infty}\left[\sum_{\tau=\xi}^{\infty}\left(\frac{1}{\tau^{\gamma}} \int_{s=\tau}^{\infty} \frac{1}{s^{\gamma+1}} d s\right)^{1 / \gamma}\right] \\
& =\frac{1}{(2 \gamma)^{1 / \gamma}} \sum_{\xi=2}^{\infty}\left[\sum_{\tau=\xi}^{\infty} \frac{1}{\tau^{2}}\right] \\
& >\frac{1}{(2 \gamma)^{1 / \gamma}} \sum_{\xi=2 \tau=\xi}^{\infty} \sum^{\infty} \frac{1}{\tau(\tau+1)}=\frac{1}{(2 \gamma)^{1 / \gamma}} \sum_{\xi=2}^{\infty} \frac{1}{\xi}=\infty .
\end{aligned}
$$

On the other hand, for a sufficiently large $T>1$, we have

$$
\delta_{1}(t, T)=\sum_{s=T}^{t-1} \frac{\left[e_{-p / a}\left(s, t_{0}\right)\right]^{1 / \gamma}}{a^{1 / \gamma}(s)}>\frac{1}{2^{1 / \gamma}} \sum_{s=T}^{t-1} \frac{1}{s} \longrightarrow \infty .
$$

So there exists $T^{*}>T$ such that $\delta_{1}(s, T)>1$ for $t \in\left[T^{*}, \infty\right)_{\mathbb{Z}}$. Let $\rho(t)=t^{\gamma}, \eta(t)=0$ in (38). Then by the inequality $(t+1)^{\gamma}-$ $t^{\gamma} \leq \gamma(t+1)^{\gamma-1}<\gamma 2^{\gamma-1} t^{\gamma-1}, t \geq T^{*}$, we obtain

$$
\begin{aligned}
& \lim _{t \rightarrow \infty} \sup \left\{\sum_{s=T}^{t-1}\left\{L \frac{q(s) \rho(s)}{e_{-p / a}\left(s+1, t_{0}\right)}-\left[\frac{r(s)(\rho(s+1)-\rho(s))}{(\gamma+1) r^{1 /(\gamma+1)}(s) \rho^{\gamma /(\gamma+1)}(s) \delta_{1}^{\gamma /(\gamma+1)}(s, T)}\right]^{\gamma+1}\right\}\right\} \\
& =\lim _{t \rightarrow \infty} \sup \left\{\sum_{s=T}^{T^{*}}\left\{L \frac{q(s) \rho(s)}{e_{-p / a}\left(s+1, t_{0}\right)}-\left[\frac{r(s)(\rho(s+1)-\rho(s))}{(\gamma+1) r^{1 /(\gamma+1)}(s) \rho^{\gamma /(\gamma+1)}(s) \delta_{1}^{\gamma /(\gamma+1)}(s, T)}\right]^{\gamma+1}\right\}\right. \\
& \left.+\sum_{s=T^{*}}^{t-1}\left\{L \frac{q(s) \rho(s)}{e_{-p / a}\left(s+1, t_{0}\right)}-\left[\frac{r(s)(\rho(s+1)-\rho(s))}{(\gamma+1) r^{1 /(\gamma+1)}(s) \rho^{\gamma /(\gamma+1)}(s) \delta_{1}^{\gamma /(\gamma+1)}(s, T)}\right]^{\gamma+1}\right\}\right\} \\
& >\lim _{t \rightarrow \infty} \sup \left\{\sum_{s=T}^{T^{*}}\left\{L \frac{q(s) \rho(s)}{e_{-p / a}\left(s+1, t_{0}\right)}-\left[\frac{r(s)(\rho(s+1)-\rho(s))}{(\gamma+1) r^{1 /(\gamma+1)}(s) \rho^{\gamma /(\gamma+1)}(s) \delta_{1}^{\gamma /(\gamma+1)}(s, T)}\right]^{\gamma+1}\right\}\right. \\
& \left.+\sum_{s=T^{*}}^{t-1}\left[M-\left(\frac{\gamma}{\gamma+1}\right)^{\gamma+1} 2^{\gamma^{2}-1}\right] \frac{1}{s}\right\} \rightarrow \infty
\end{aligned}
$$

provided that $M>(\gamma /(\gamma+1))^{\gamma+1} 2^{\gamma^{2}-1}$. So (37) and (38) all hold, and by Corollary 11 we obtain that every solution of (69) is oscillatory or tends to zero under the condition $M>(\gamma /(\gamma+$ 1) $)^{\gamma+1} 2^{\gamma^{2}-1}$. 


\section{Acknowledgments}

The authors would like to thank the reviewers very much for their valuable suggestions on this paper.

\section{References}

[1] S. Hilger, "Analysis on measure chains-a unified approach to continuous and discrete calculus," Results in Mathematics, vol. 18, no. 1-2, pp. 18-56, 1990.

[2] M. Bohner, "Some oscillation criteria for first order delay dynamic equations," Far East Journal of Applied Mathematics, vol. 18, no. 3, pp. 289-304, 2005.

[3] B. G. Zhang and X. Deng, "Oscillation of delay differential equations on time scales," Mathematical and Computer Modelling, vol. 36, no. 11-13, pp. 1307-1318, 2002.

[4] Z.-Q. Zhu and Q.-R. Wang, "Existence of nonoscillatory solutions to neutral dynamic equations on time scales," Journal of Mathematical Analysis and Applications, vol. 335, no. 2, pp. 751762, 2007.

[5] R. P. Agarwal, M. Bohner, and S. H. Saker, "Oscillation of second order delay dynamic equations," The Canadian Applied Mathematics Quarterly, vol. 13, no. 1, pp. 1-17, 2005.

[6] E. Akin-Bohner, M. Bohner, and S. H. Saker, "Oscillation criteria for a certain class of second order Emden-Fowler dynamic equations," Electronic Transactions on Numerical Analysis, vol. 27, pp. 1-12, 2007.

[7] M. Bohner and S. H. Saker, "Oscillation of second order nonlinear dynamic equations on time scales," The Rocky Mountain Journal of Mathematics, vol. 34, no. 4, pp. 1239-1254, 2004.

[8] L. Erbe, A. Peterson, and S. H. Saker, "Oscillation criteria for second-order nonlinear delay dynamic equations," Journal of Mathematical Analysis and Applications, vol. 333, no. 1, pp. 505522, 2007.

[9] S. R. Grace, R. P. Agarwal, M. Bohner, and D. O'Regan, "Oscillation of second-order strongly superlinear and strongly sublinear dynamic equations," Communications in Nonlinear Science and Numerical Simulation, vol. 14, no. 8, pp. 3463-3471, 2009.

[10] Y. Şahiner, "Oscillation of second-order delay differential equations on time scales," Nonlinear Analysis: Theory, Methods \& Applications, vol. 63, no. 5-7, pp. e1073-e1080, 2005.

[11] S. H. Saker, "Oscillation of nonlinear dynamic equations on time scales," Applied Mathematics and Computation, vol. 148, no. 1, pp. 81-91, 2004.

[12] B. Jia, L. Erbe, and A. Peterson, "New comparison and oscillation theorems for second-order half-linear dynamic equations on time scales," Computers \& Mathematics with Applications, vol. 56, no. 10, pp. 2744-2756, 2008.

[13] Z. Han, S. Sun, and B. Shi, "Oscillation criteria for a class of second-order Emden-Fowler delay dynamic equations on time scales," Journal of Mathematical Analysis and Applications, vol. 334, no. 2, pp. 847-858, 2007.

[14] S. R. Grace, M. Bohner, and R. P. Agarwal, "On the oscillation of second-order half-linear dynamic equations," Journal of Difference Equations and Applications, vol. 15, no. 5, pp. 451-460, 2009.

[15] M. Bohner and S. H. Saker, "Oscillation criteria for perturbed nonlinear dynamic equations," Mathematical and Computer Modelling, vol. 40, no. 3-4, pp. 249-260, 2004.
[16] L. Erbe, A. Peterson, and S. H. Saker, "Oscillation criteria for second-order nonlinear dynamic equations on time scales," Journal of the London Mathematical Society, vol. 67, no. 3, pp. 701-714, 2003.

[17] L. Erbe, A. Peterson, and S. H. Saker, "Kamenev-type oscillation criteria for second-order linear delay dynamic equations," Dynamic Systems and Applications, vol. 15, no. 1, pp. 65-78, 2006.

[18] S. H. Saker, "Oscillation criteria of second-order half-linear dynamic equations on time scales," Journal of Computational and Applied Mathematics, vol. 177, no. 2, pp. 375-387, 2005.

[19] S. H. Saker, "Oscillation of second-order nonlinear neutral delay dynamic equations on time scales," Journal of Computational and Applied Mathematics, vol. 187, no. 2, pp. 123-141, 2006.

[20] M. Huang and W. Feng, "Forced oscillation of second order nonlinear dynamic equations on time scales," Electronic Journal of Qualitative Theory of Differential Equations, vol. 2008, no. 36, pp. 1-13, 2008.

[21] L. Erbe, A. Peterson, and S. H. Saker, "Asymptotic behavior of solutions of a third-order nonlinear dynamic equation on time scales," Journal of Computational and Applied Mathematics, vol. 181, no. 1, pp. 92-102, 2005.

[22] L. Erbe, A. Peterson, and S. H. Saker, "Hille and Nehari type criteria for third-order dynamic equations," Journal of Mathematical Analysis and Applications, vol. 329, no. 1, pp. 112131, 2007.

[23] L. Erbe, A. Peterson, and S. H. Saker, "Oscillation and asymptotic behavior of a third-order nonlinear dynamic equation," The Canadian Applied Mathematics Quarterly, vol. 14, no. 2, pp. 129-147, 2006.

[24] L. Erbe, T. S. Hassan, and A. Peterson, "Oscillation of third order functional dynamic equations with mixed arguments on time scales," Journal of Applied Mathematics and Computing, vol. 34, no. 1-2, pp. 353-371, 2010.

[25] T. S. Hassan, "Oscillation of third order nonlinear delay dynamic equations on time scales," Mathematical and Computer Modelling, vol. 49, no. 7-8, pp. 1573-1586, 2009.

[26] B. Karpuz, "Unbounded oscillation of higher-order nonlinear delay dynamic equations of neutral type with oscillating coefficients," Electronic Journal of Qualitative Theory of Differential Equations, vol. 2009, no. 34, pp. 1-14, 2009.

[27] B. Karpuz, "Asymptotic behaviour of bounded solutions of a class of higher-order neutral dynamic equations," Applied Mathematics and Computation, vol. 215, no. 6, pp. 2174-2183, 2009.

[28] Z.-H. Yu and Q.-R. Wang, "Asymptotic behavior of solutions of third-order nonlinear dynamic equations on time scales," Journal of Computational and Applied Mathematics, vol. 225, no. 2, pp. 531-540, 2009.

[29] Z. Han, T. Li, S. Sun, and F. Cao, "Oscillation criteria for third order nonlinear delay dynamic equations on time scales," Annales Polonici Mathematici, vol. 99, no. 2, pp. 143-156, 2010.

[30] Z. Han, T. Li, S. Sun, and C. Zhang, "Oscillation behavior of third-order neutral Emden-Fowler delay dynamic equations on time scales," Advances in Difference Equations, vol. 2010, Article ID 586312, 23 pages, 2010.

[31] L. Erbe, T. S. Hassan, and A. Peterson, "Oscillation of third order nonlinear functional dynamic equations on time scales," Differential Equations and Dynamical Systems, vol. 18, no. 1-2, pp. 199-227, 2010. 
[32] S. R. Grace, J. R. Graef, and M. A. El-Beltagy, "On the oscillation of third order neutral delay dynamic equations on time scales," Computers \& Mathematics with Applications, vol. 63, no. 4, pp. 775-782, 2012.

[33] Y. Sun, Z. Han, Y. Sun, and Y. Pan, "Oscillation theorems for certain third order nonlinear delay dynamic equations on time scales," Electronic Journal of Qualitative Theory of Differential Equations, vol. 2011, no. 75, pp. 1-14, 2011.

[34] S. H. Saker, "Oscillation of third-order functional dynamic equations on time scales," Science China. Mathematics, vol. 54, no. 12, pp. 2597-2614, 2011.

[35] S. H. Saker, "On oscillation of a certain class of third-order nonlinear functional dynamic equations on time scales," Bulletin Mathématique de la Société des Sciences Mathématiques de Roumanie, vol. 54, no. 4, pp. 365-389, 2011.

[36] R. Agarwal, M. Bohner, and A. Peterson, "Inequalities on time scales: a survey," Mathematical Inequalities \& Applications, vol. 4, no. 4, pp. 535-557, 2001.

[37] M. Bohner and A. Peterson, Dynamic Equations on Time Scales: An Introduction with Applications, Birkhäuser, Boston, Mass, USA, 2001.

[38] G. H. Hardy, J. E. Littlewood, and G. Pólya, Inequalities, Cambridge Mathematical Library, Cambridge University Press, Cambridge, UK, 1988. 


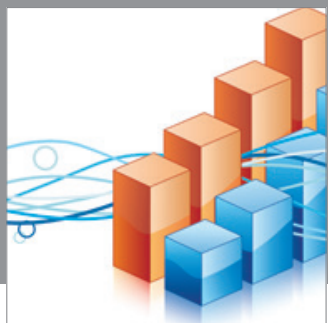

Advances in

Operations Research

mansans

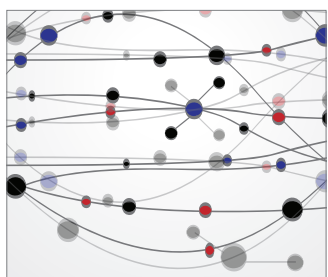

The Scientific World Journal
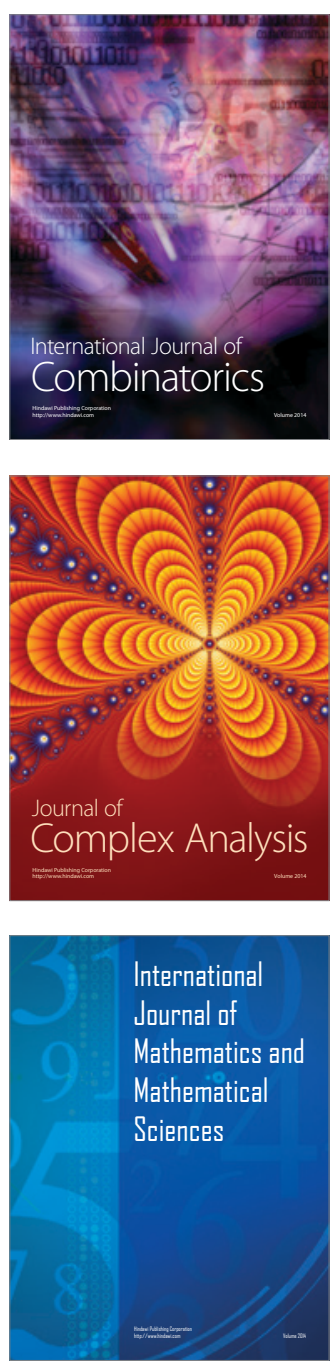
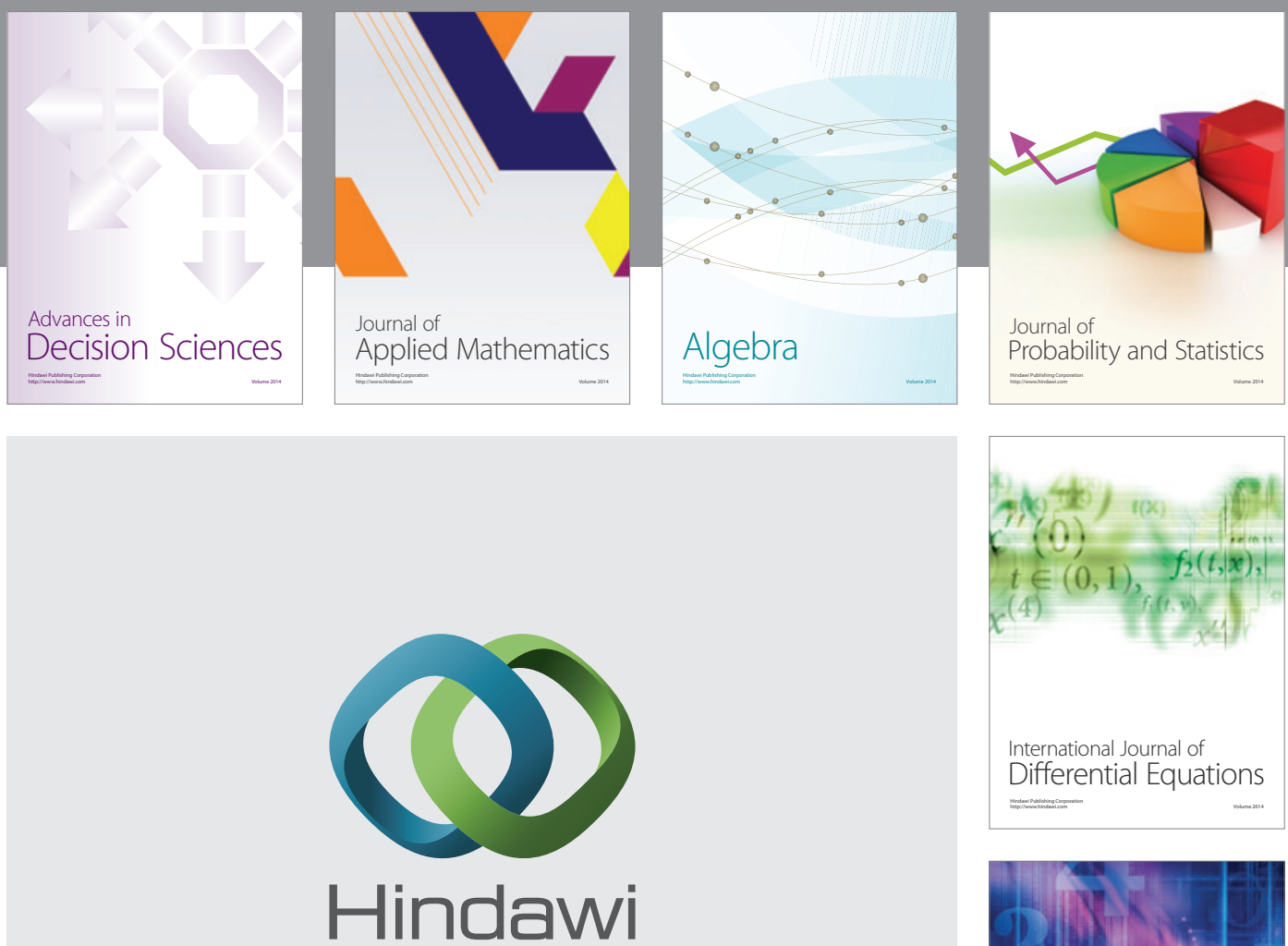

Submit your manuscripts at http://www.hindawi.com
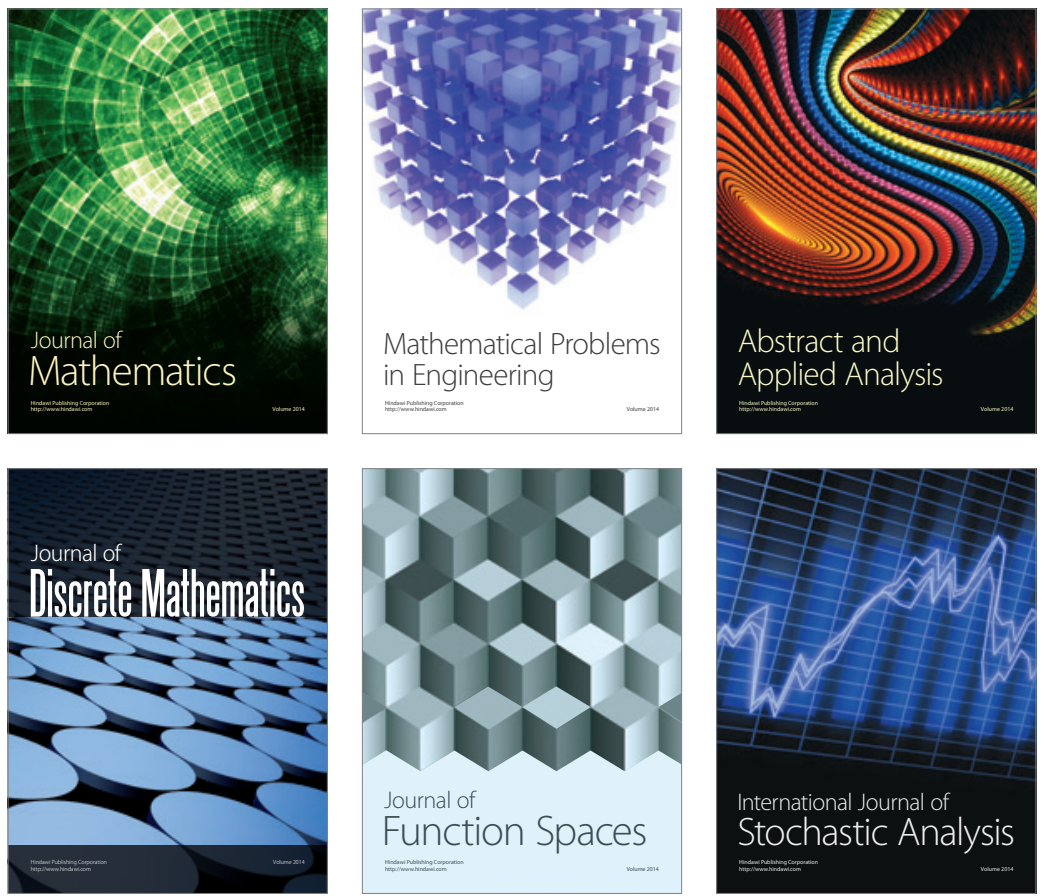

Journal of

Function Spaces

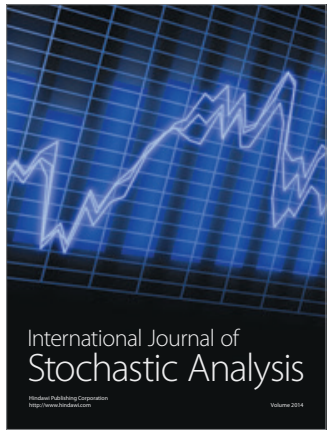

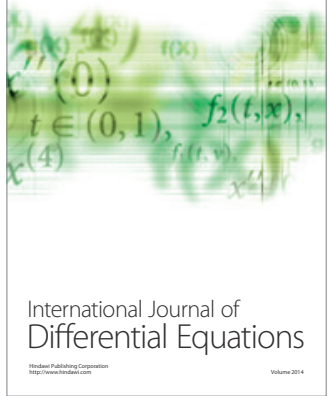
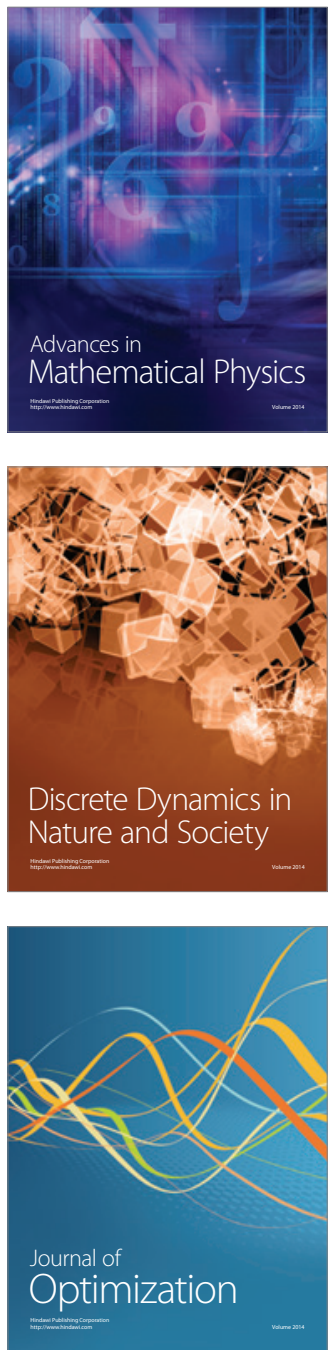